\title{
Group-Based Approach to Predictive Delay Model Based on Incremental Queue Accumulations for Adaptive Traffic Control Systems
}

\author{
Seunghyeon Lee and S.C. Wong \\ Department of Civil Engineering, The University of Hong Kong, Pokfulam Road, Hong Kong, China
}

\begin{abstract}
In this study, we develop a mathematical framework to estimate lane-based incremental queue accumulations with group-based variables and a predictive model of lane-based control delay. Our objective is to establish the rolling horizon approach to lane-based control delay for group-based optimization of signal timings in adaptive traffic control systems. The challenges involved in this task include identification of the most appropriate incremental queue accumulations based on group-based variables for individual lanes to the queueing formation patterns and establishment of the rolling horizon procedure for predicting the future components of lane-based incremental queue accumulations in the time windows. For lane-based estimation of incremental queue accumulations, temporal and spatial information were collected on the basis of estimated lane-based queue lengths from our previous research to estimate lane-based incremental queue accumulations. We interpret the given signal plan as group-based variables, including the start and duration of the effective green time and the cycle time. Adjustment factors are defined to identify the characteristics of the control delay in a specific cycle and to clarify the relationship between group-based variables and the temporal information of queue lengths in the proposed estimation method. We construct the rolling horizon procedure based on Kalman filters with appropriate time windows. Lane-based queue lengths at an inflection point and adjustment factors in the previous cycle are used to estimate the adjustment factors, arrival rates, and discharge rates in the next cycle, in which the predictive computation is performed in the current cycle. In the simulations sets and the case study, the proposed model is robust and accurate for estimation of lane-based control delay under a wide range of traffic conditions. Adjustment factors play a significant role in increasing the accuracy of the proposed model and in classifying queueing patterns in a specific cycle. The Kalman filters enhance the accuracy of the predictions by minimizing the error terms caused by the fluctuation in traffic flow.
\end{abstract}

Keywords: Incremental queue accumulations, Lane-based control delay, Kalman filter, Adjustment factors, Rolling horizon approach

\section{INTRODUCTION}

Traffic signals are the most effective means to control conflicting traffic flows at isolated signalized junctions. Adaptive traffic control systems (ATCS), in which signal settings are established in real time on the basis of the most up-to-date traffic detector information, have shown better performance than fixed-time controls in many places, despite their higher construction and operation costs. To construct adaptive traffic control logics, estimation of a control delay incurred by queued vehicles in both long-term and short-term analysis periods is required for the control system. The great part of the control delay, which is commonly used as the performance index in ATCS, was derived from Webster's theories and equations (Webster, 1958), stochastic queueing theory (Saaty, 1961), queued vehicular delay (Miller, 1963), and time-dependent delay formula (Akçelik, 1980).

The estimation methods of the control delay have been integrated with major queue estimation methods, including the conservation equation (Lindley, 1952) and the shockwave theory (Lighthill and Whitham, 1955; Richards, 1965). Following the aforementioned cornerstones for the development of a control delay and queue estimation methods, diverse delay estimation methods have been developed, including both deterministic and stochastic characteristics of a control delay. The 
fundamental concept of the control delay, the incremental queue accumulation (IQA), has been continuously developed in the U.S. Highway Capacity Manual (TRB, 2010) to improve the accuracy and robustness of the delay estimation methods.

Because of its simplicity, a stage-based method defined by a predetermined sequence of stages and intergreen periods is currently the most common technique used to construct signal timing plans at isolated signalized junctions and networks. Allsop $(1971,1972)$ constructed mathematical programs to minimize delay and maximize capacity at isolated junctions. To pursue a more flexible structure of signal timings and greater applicability to diverse urban traffic and road geometric conditions, a group-based method, in which signal settings are defined by the start and duration of green signal groups, was introduced by Heydecker and Dudgeon (1987) and Heydecker (1992). This method included a procedure to simultaneously optimize the structure of intergreen periods, a cycle length, and a signal sequence. Silcock (1997) crystallized a group-based optimization method at isolated signalized junctions to specify a detailed mathematical framework for the procedure. Wong (1996, 1997) extended the group-based method for area traffic controls with derivatives of the performance index (Wong, 1995). Wong and Wong (2003a, 2003b) and Wong et al. (2006) devised a lane-based approach to integrate a group-based optimization method with the geometric design of lane markings. The notable advantage of the group-based method is the flexibility of signal timings to optimize both signal timings and a cycle structure without the use of a predefined set of stages and a signal sequence.

For adaptive control logics, a two-phase heuristic signal control algorithm, which was one of the first contributions to real-time responsive signal control systems, was introduced by Dunne and Potts (1964) and Green (1967). The fundamental concept of real-time responsive signal control systems was specialized into traffic-actuated control and ATCS. Morris and Pak-Poy (1967) and Gordon et al. (2005) proposed traffic-actuated control systems that use simple logic to extend and terminate the current state of a signal controller on the basis of real-time traffic information collected from stop-line or upstream detectors. Despite its simplicity and relatively widespread availability in the field, these systems have several challenges, such as a rigid cycle structure, reactive logics to only current traffic conditions, an absence of optimal solutions for long-term or large-scale networks, and a lack of effectiveness in oversaturated traffic situations. To overcome these critical issues, simple responsive signal control systems have evolved into a more comprehensive and complicated form of ATCS with the concept of long-term and short-term control delay as the performance index. Miller (1963) developed an algorithm to operate a simple traffic-actuated logic according to vehicular delay as a binary choice approach, which is one of the most common concepts in the ATCS. Smith (1979a, 1979b, 1981), Smith and Ghali (1990), Smith and Van Vuren (1993), Smith (1983), and Smith and Mounce (2015) continuously developed one of the most important theoretical philosophies of ATCS - the P0 signal control policy - over several decades. Following both Miller's algorithm and the P0 signal control policy, the store and forward modeling approach, which is the foundation of mathematical models to optimize signal timings in real time, was developed and applied in diverse traffic conditions by Gazis (1964), Gazis and Potts (1965), Grafton and Newell (1967), Ross et al. (1971), Rosdolsky (1973), D'ans and Gazis (1976), Bang and Nilsson (1976), Michalopoulos and Stephanopoulos (1977a, 1977b), and Aboudolas et al. (2009). The methods to construct a cyclestructure according to a diverse level of temporal and spatial scales of control delay have been installed in many places such as PRODYN (Farges et al., 1983), SCOOT (Hunt et al., 1981), OPAC (Gartner, 1983), RHODES (Mirchandani and Head, 2001), ACS-Lite (Luyanda et al., 2003), and CRONOS (Boillot et al., 2006). They are based on the store and forward philosophy combined with a diverse rolling horizon predictive model for the control delay and turning movements, dynamic programming methods, and additional special features. In addition to the adaptive control approaches mentioned above, Varaiya (2013) proposed the max pressure traffic signal control policy at both isolated signalized junctions and networks. The max pressure control only requires turning flows and queue lengths at each intersection and adjacent intersections for network optimization without prior knowledge of traffic demands. To reduce the computational demands in a real-time calculation of signal timings with flexible reasonable analysis periods and good performance, Smith (2011), Ge and Zhou (2012), Han et al. (2014), and Han and Gayah (2015) consistently studied continuum signal 
models to approximate traffic dynamics. ATCS take full advantage of their use of sensor technologies to update traffic patterns in real time and to pursue flexibility in adjusting the control strategies to cater to these most up-to-date traffic patterns in an adaptive manner according to estimates of the control delay in real time.

To introduce a group-based method in ATCS, Lee et al., (2015a) took the first step toward estimation of lane-to-lane turning flows, which is the essential input for construction of adaptive signal control logic on a real-time basis. Lee et al. (2015b) then developed a real-time estimation method of lane-based vertical queue lengths based on discriminant models and estimated downstream arrivals to estimate cycle-delay for adaptive control logics. In this study, we devise a method with which to estimate lane-based control delay on the basis of the required temporal and spatial information to calculate real-time arrival and discharge rates in IQAs obtained from the aforementioned lane-based queue-length estimation method. For the predictive procedure, we used Kalman filters to predict temporal and spatial factors in future IQAs and produce the most appropriate time windows for a rolling horizon procedure.

\section{METHODS}

This section shows the formulation of the proposed method, including group-based variables, lane-based IQAs, and lane-based delay predictive models. The proposed data-processing method for the lane-based control delay predictive model based on IQAs and group-based methods is illustrated in Figure 1.

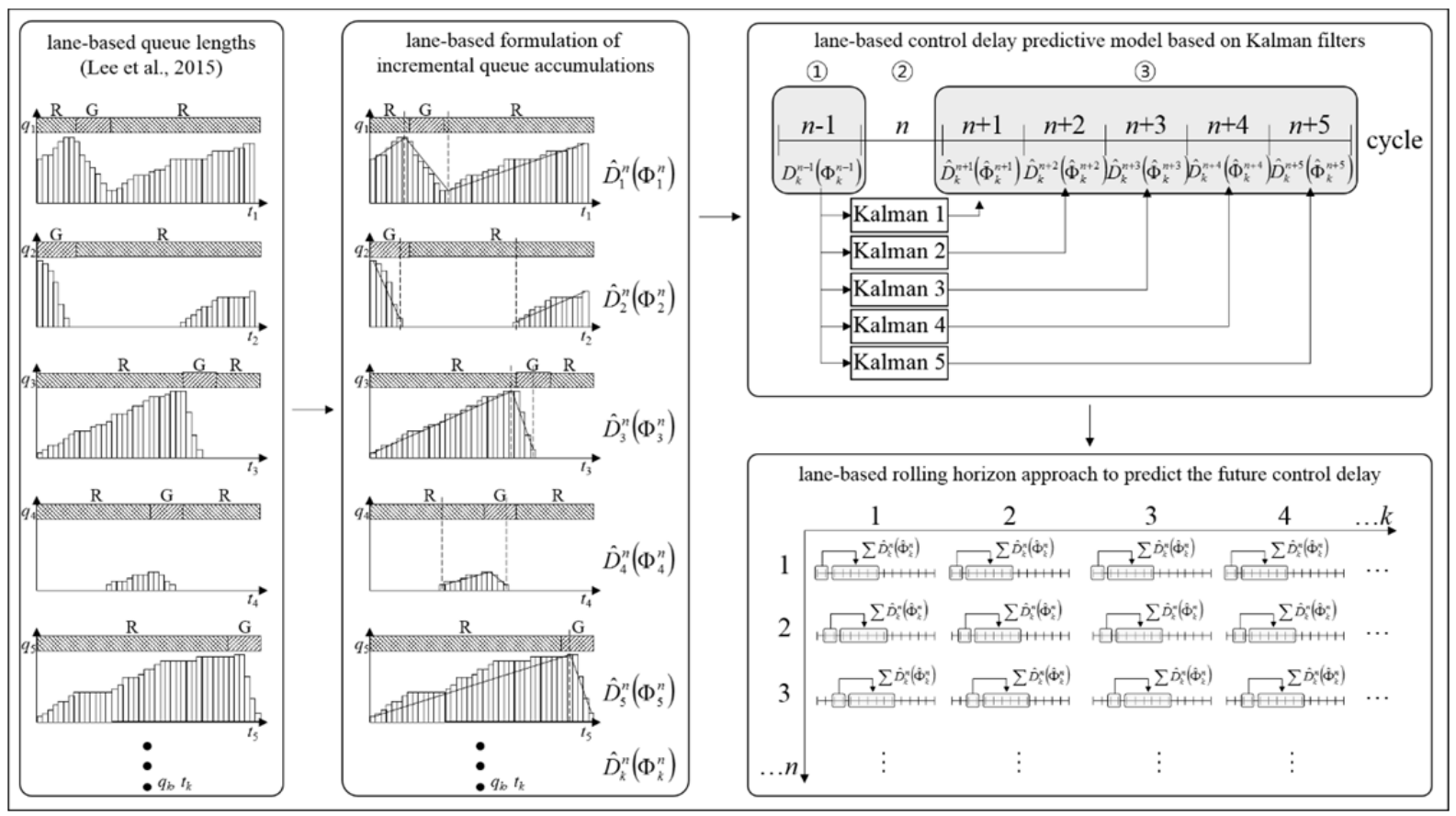

Figure 1. Data processing method for lane-based control delay predictive models.

In the first step in Figure 1, real-time queue lengths in individual lanes are estimated by the lane-based queue length estimation method developed in Lee et al. (2015b). We collect the temporal and spatial information of lane-based queue lengths to formulate lane-based IQAs in real time from the estimated queue lengths. In the second step, the polygons in IQAs in lane $k$ on the $n$th cycle, $\hat{D}_{k}^{n}\left(\Phi_{k}^{n}\right)$, are specified by group-based variables, including the start and duration of the green time for each signal group and the cycle time, and estimated temporal and spatial information of the queued vehicles with adjustment factors, used to define the queueing formation patterns in each lane on each cycle in this paper. These polygons show the control delay in each lane on the $n$th cycle and they are estimated through the proposed mathematical framework in this paper. After estimating the 
control delay in individual lanes, these estimates are used in the next step to predict the future components of the polygons by a recursive predictive procedure based on Kalman filters. In the predictive model, the established formulations for the control delay in the $(n-1)$ th cycle (section in Figure 1), $\hat{D}_{k}^{n-1}\left(\Phi_{k}^{n-1}\right)$, are used to predict the components of future IQAs, $\hat{D}_{k}^{n+1}\left(\hat{\Phi}_{k}^{n+1}\right)$, $\hat{D}_{k}^{n+2}\left(\hat{\boldsymbol{\Phi}}_{k}^{n+2}\right), \hat{D}_{k}^{n+3}\left(\hat{\boldsymbol{\Phi}}_{k}^{n+3}\right), \hat{D}_{k}^{n+4}\left(\hat{\boldsymbol{\Phi}}_{k}^{n+4}\right)$, and $\hat{D}_{k}^{n+5}\left(\hat{\boldsymbol{\Phi}}_{k}^{n+5}\right)$, for the appropriate time windows using each different Kalman filter (section in Figure 1) to avoid irregular and over-sensitive predictions. The calculation time (section in Figure 1) is provided in the current $n$th cycle. In the last step, the proposed predictive model is used to construct a lane-based rolling-horizon approach to predict the future control delay on the basis of predicted temporal and spatial information of the queued vehicles in individual lanes and adjustment factors within the corresponding time windows. The control delay at the target intersection, $\sum_{k=1}^{K} \hat{D}_{k}^{n+1}\left(\hat{\Phi}_{k}^{n+1}\right)$, is calculated on the $n$th cycle on the basis of the data collected in the $(n-1)$ th cycle. This data processing is performed on every cycle in each individual lane to estimate the future variables for prediction of the future patterns of the control delay. below.

The definitions of the common indices, parameters, and variables used in this study are given

$\begin{array}{ll}K & \text { number of lanes } \\ N & \text { number of cycles } \\ T & \text { number of time steps in a cycle } \\ q_{k}^{n}(t) & \text { number of queued vehicles in lane } k \text { at time } t \text { in the } n \text {th cycle } \\ \theta_{k}^{n} & \text { time from cycle origin to start of actual green for control group } l \text { divided by cycle time in } \\ \phi_{k}^{n} & \text { the } n \text {th cycle } \\ \zeta^{n} & \text { duration of actual green for control group } l \text { divided by cycle time in the } n \text {th cycle }\end{array}$

$K, N$, and $T$ are the common indices for the several steps in this paper, and $q_{k}^{n}(t)$ represents the input data, which are the final output of the lane-based queue estimation models introduced by Lee et al. (2015b). $\theta_{k}^{n}, \phi_{k}^{n}$, and $\zeta^{n}$ are commonly used temporal group-based variables and introduced by Heydecker and Dudgeon (1987).

\subsection{Real-time estimation method of lane-based queue lengths}

To account for lane-changing behavior and counting errors, the figure for estimating downstream arrivals, $\hat{a}_{\mathrm{d}, k}^{n}(t)$, is used. The modified conservation equation is therefore given as

$$
q_{k}^{n}(t)= \begin{cases}\max \left\{x_{k}^{n}(t) q_{k}^{n-1}(T)+\hat{a}_{\mathrm{d}, k}^{n}(t)-i_{\mathrm{d}, k}^{n}(t), 0\right\}, & \text { for } t=1 \\ \max \left\{q_{k}^{n}(t-1)+\hat{a}_{\mathrm{d}, k}^{n}(t)-i_{\mathrm{d}, k}^{n}(t), 0\right\} . & \text { for } t \neq 1\end{cases}
$$

Detailed explanations of the overall process to estimate queue lengths are given in Lee et al. (2015b). To account for possible counting and passage errors, the number of queued vehicles in lane $k$ at time $t$ in the $n$th cycle, $q_{k}^{n}(t)$, is the maximum value between the conservation equation and zero. The first equation is used for the start time of each cycle, and the second for other time intervals. $q_{k}^{n-1}(T)$ is the number of queued vehicles in lane $k$ at the last time point $T$ in the $(n-1)$ th cycle, and $x_{k}^{n}(t)$ is the output of the discriminant models. For example, if there is a residual queue in lane $k$ at time $T$ in the $(n-1)$ th cycle, then $x_{k}^{n}(t)=1$, and otherwise 0 . Furthermore, let $J$ be the set of upstream 
lanes, and let $K$ the set of downstream lanes. $i_{\mathrm{d}, k}^{n}(t)$ is the downstream discharge according to the binary impulse memory from the downstream detector in lane $k$ at time $t$ in the $n$th cycle. The number of queued vehicles in each lane is recursively estimated on the basis of these equations in real time. These estimates are then used to establish a signal plan for the current cycle and calculate the stagebased pressure.

\subsection{Lane-based IQAs based on group-based variables}

The real-time estimated number of queued vehicles is used to design the polygons in the IQAs. We can simultaneously collect the queue lengths and the time in which the queue lengths drastically change from the previous step. The $\mathrm{x}$ - and $\mathrm{y}$-coordinates of the polygons are based on these collected spatial and temporal data as described by queue lengths, group-based variables, and adjustment factors. The concept of lane-based IQAs with the aforementioned variables is illustrated in Figure 2.

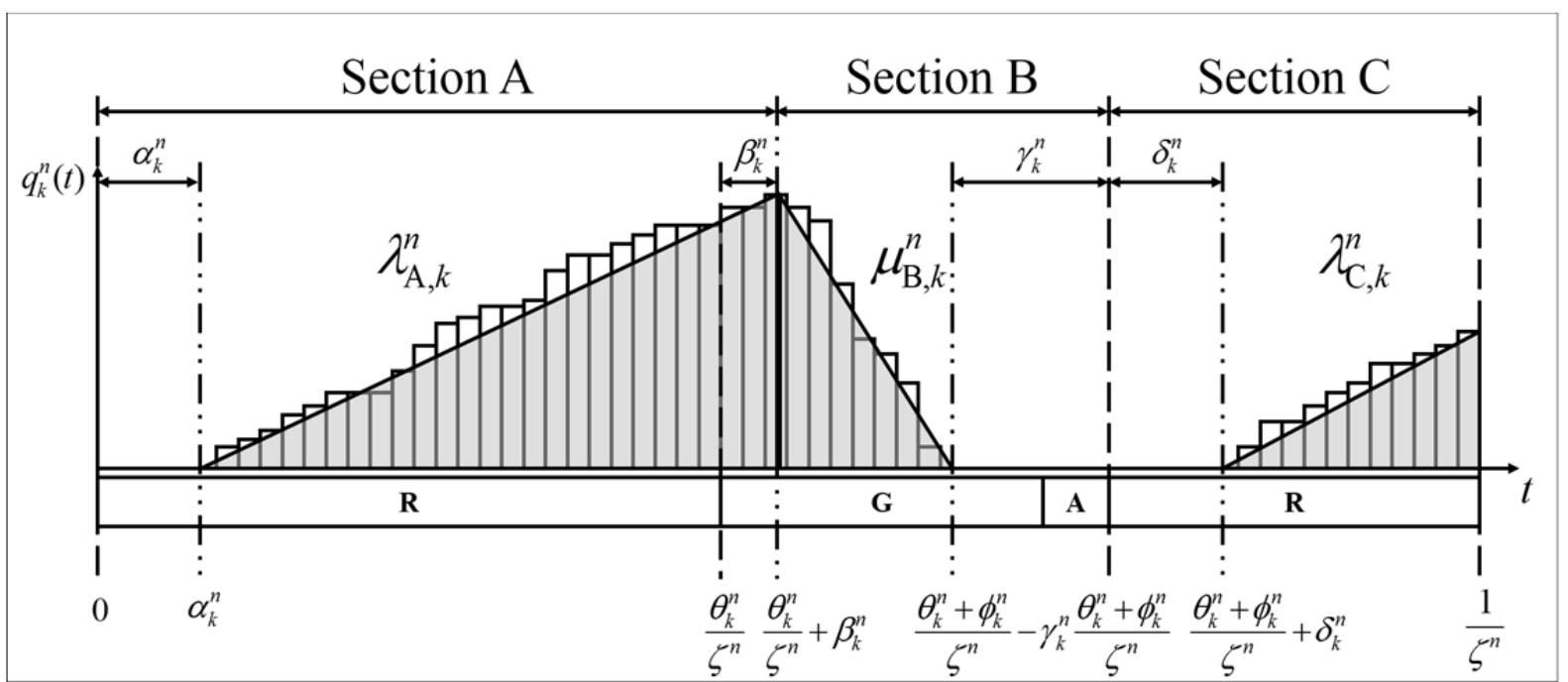

Figure 2. Lane-based incremental queue accumulations.

In Figure 2, the y-coordinate is the queue length, and the $\mathrm{x}$-coordinate is the time in seconds. The cycle time in the $n$th cycle is $1 / \zeta^{n}$, and the start and end of the effective green time in lane $k$ in the $n$th cycle are $\theta_{k}^{n} / \zeta^{n}$ and $\left(\theta_{k}^{n}+\phi_{k}^{n}\right) / \zeta^{n}$, respectively. We use the adjustment factor, $\alpha_{k}^{n}$, to consider the period during which the queue length in lane $k$ is zero during the red time before the effective green time in the $n$th cycle. Similarly, $\beta_{k}^{n}$ considers the period in which the queue length in lane $k$ is continuously increasing, even if the current state of a signal controller is the green time in the $n$th cycle. The number of time slots during which lane $k$ is empty during the effective green time in the $n$th cycle is captured by the factor, $\gamma_{k}^{n}$, as a negative number, whereas $\gamma_{k}^{n}$ becomes a positive number if the queue length is still discharging after the effective green time. $\delta_{k}^{n}$ describes the number of time units during which there are no queues in lane $k$ during the red time after the effective green time in the $n$th cycle. $\lambda_{\mathrm{A}, k}^{n}$ and $\lambda_{\mathrm{C}, k}^{n}$ are the queue rates in lane $k$ in the $n$th cycle in sections $\mathrm{A}$ and $\mathrm{C}$, respectively. $\mu_{\mathrm{B}, k}^{n}$ is the queue rate in lane $k$ in the $n$th cycle in section $\mathrm{B}$.

\subsubsection{Section A}

For section $\mathrm{A}, \lambda_{\mathrm{A}, k}^{n}$ can be categorized into four formulations according to the traffic conditions described in Figure 3. If queued vehicles remain in lane $k$ in the $n$th cycle, which is generally considered to be oversaturated traffic conditions, or the queue length in lane $k$ starts to build up from the start of a cycle, $\alpha_{k}^{n}$ becomes zero. In contrast, $\alpha_{k}^{n}$ is not set as zero if the queue length in 
lane $k$ is built up in the middle of the red periods before the effective green time in the $n$th cycle. In addition, if the queue length is still built up after the start of the effective green time, $\beta_{k}^{n}$ becomes the number of time slots, in which the arrival rate is positive; otherwise, $\beta_{k}^{n}$ is set as zero.

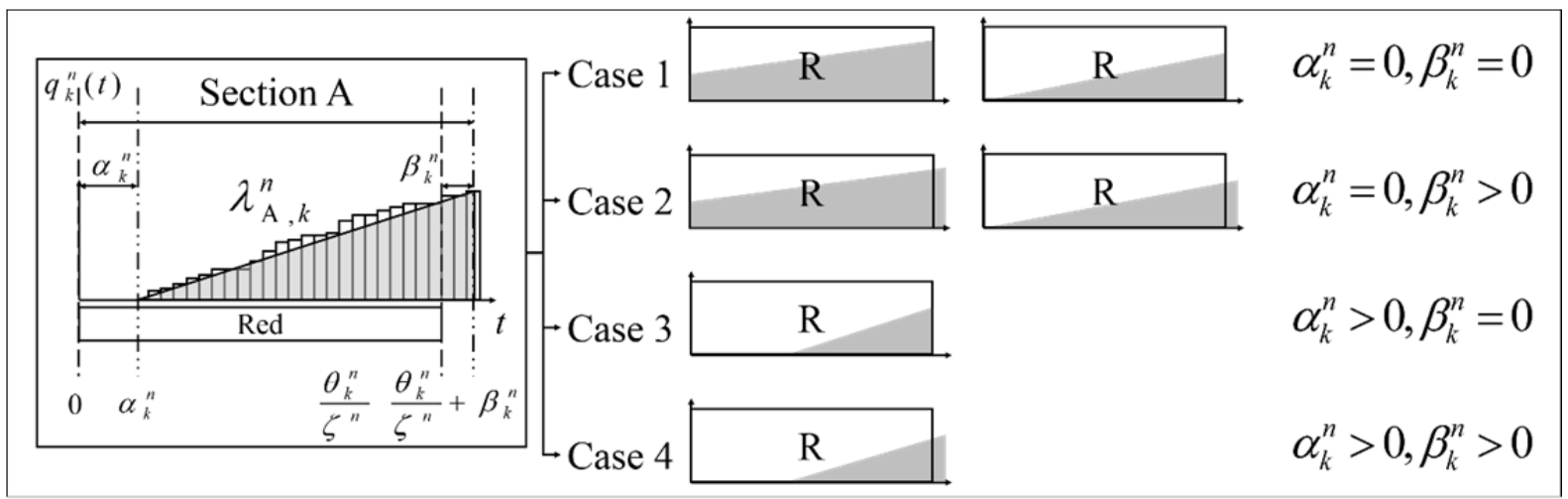

Figure 3. Four queueing formation patterns on Section A.

These adjustment factors are included in the arrival rate and the formulation for the control delay on section $\mathrm{A}$ in the following equations.

$$
\begin{aligned}
\lambda_{\mathrm{A}, k}^{n}= & \frac{\hat{q}_{k}^{n}\left(\frac{\theta_{k}^{n}}{\zeta^{n}}+\beta_{k}^{n}\right)-\hat{q}_{k}^{n}\left(\alpha_{k}^{n}\right)}{\frac{\theta_{k}^{n}}{\zeta^{n}}+\beta_{k}^{n}-\alpha_{k}^{n}} \\
D_{\mathrm{A}, k}^{n}= & \frac{1}{2}\left[\left\{2 \hat{q}_{k}^{n}\left(\alpha_{k}^{n}\right)+\lambda_{\mathrm{A}, k}^{n}\left(\frac{\theta_{k}^{n}}{\zeta^{n}}+\beta_{k}^{n}-\alpha_{k}^{n}\right)\right\}\left(\frac{\theta_{k}^{n}}{\zeta^{n}}+\beta_{k}^{n}-\alpha_{k}^{n}\right)\right]
\end{aligned}
$$

The arrival rate during the red time before the effective green time, $\lambda_{\mathrm{A}, k}^{n}$, is described as a general formula to calculate a slope between two points, $\left(\alpha_{k}^{n}, \hat{q}_{k}^{n}\left(\alpha_{k}^{n}\right)\right),\left(\frac{\theta_{k}^{n}}{\zeta^{n}}+\beta_{k}^{n}, \hat{q}_{k}^{n}\left(\frac{\theta_{k}^{n}}{\zeta^{n}}+\beta_{k}^{n}\right)\right)$ in Equation (2). In Equation (3), which is related with a given trapezoid, the control delay on section A is computed. The first term in a brace is the number of queued vehicles in lane $k$ at $\alpha_{k}^{n}, \hat{q}_{k}^{n}\left(\alpha_{k}^{n}\right)$ plus the number of queued vehicles in lane $k$ at $\frac{\theta_{k}^{n}}{\zeta^{n}}+\beta_{k}^{n}, \hat{q}_{k}^{n}\left(\alpha_{k}^{n}\right)+\lambda_{\mathrm{A}, k}^{\mathrm{n}}\left(\frac{\theta_{k}^{n}}{\zeta^{n}}+\beta_{k}^{n}-\alpha_{k}^{n}\right)$. The term in the second brace is a duration, $\frac{\theta_{k}^{n}}{\zeta^{n}}+\beta_{k}^{n}-\alpha_{k}^{n}$, in which the arrival rate is a positive in section A. To maintain the reliable and reasonable predicted vales at the forecasting steps, we departmentalize the components in the formulation of equations instead directly using and forecasting the queue lengths to calculate the control delay in the future cycles.

\subsubsection{Section $B$}

For section $\mathrm{B}$, we classify $\mu_{\mathrm{B}, k}^{n}$ into six queueing formation patterns depending on the traffic conditions described in Figure 4. If the number of queued vehicles is still built up after the start of the effective green time, $\beta_{k}^{n}$ becomes the number of time slots, in which the arrival rate is positive; 
otherwise, $\beta_{k}^{n}$ is set as zero. We set $\gamma_{k}^{n}$ as the adjustment factor in the end of the effective green time. If the number of seconds during which lane $k$ is empty during the effective green time is larger than 0 , $\gamma_{k}^{n}$ is set as that seconds as a negative. If the queued vehicles are still departing from lane $k, \gamma_{k}^{n}$ is set as the number of seconds until discharge stops after the effective green time in the $n$th cycle; otherwise, $\gamma_{k}^{n}$ is set as zero.

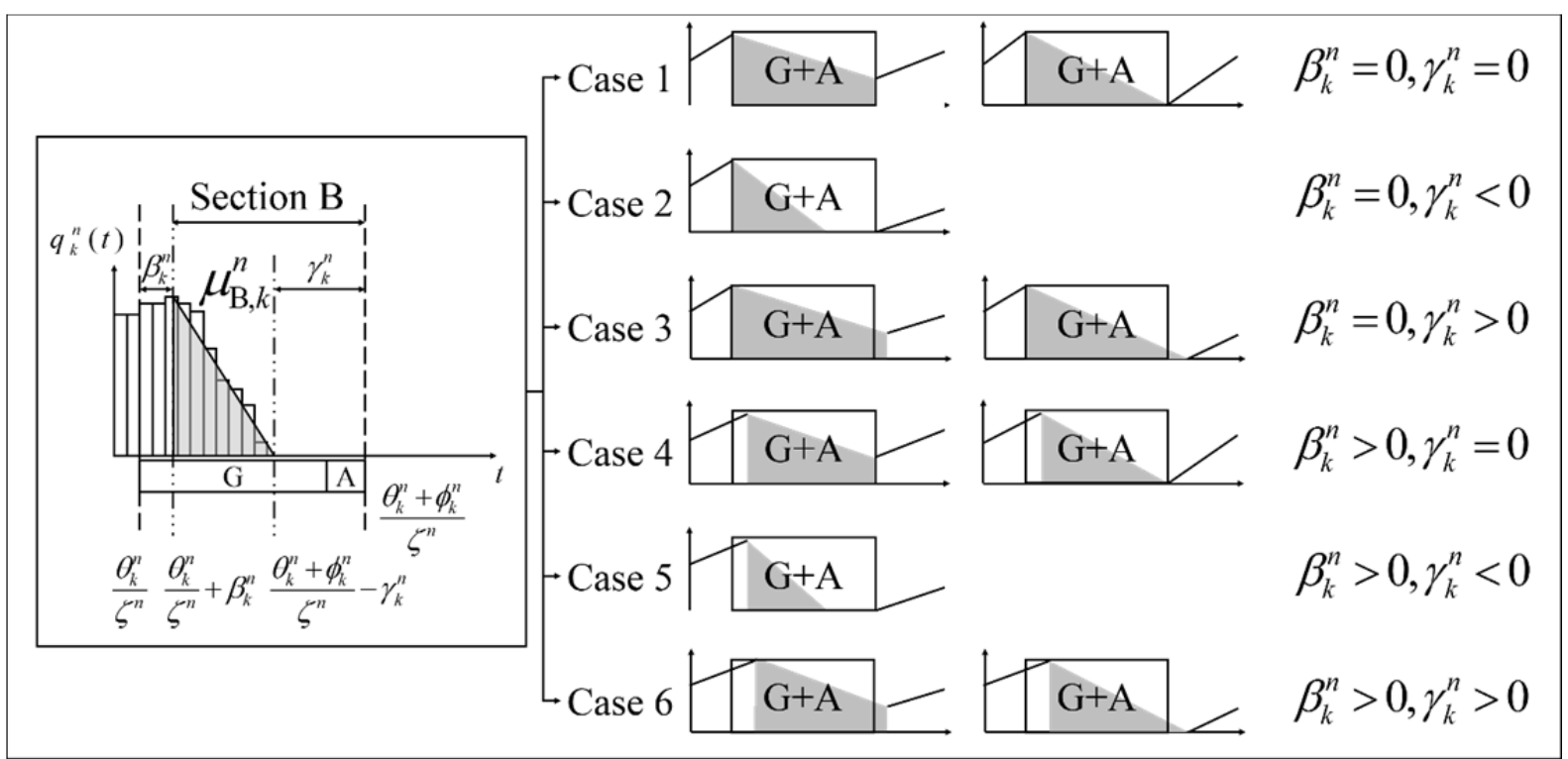

Figure 4. Six queueing formation patterns on Section B.

$\beta_{k}^{n}$ and $\gamma_{k}^{n}$ are included in the queue rate and the mathematical expression for the control delay on section $\mathrm{B}$ in the following equations.

$\mu_{\mathrm{B}, k}^{n}=\frac{\hat{q}_{k}^{n}\left(\frac{\theta_{k}^{n}+\phi_{k}^{n}}{\zeta^{n}}+\gamma_{k}^{n}\right)-\hat{q}_{k}^{n}\left(\frac{\theta_{k}^{n}}{\zeta^{n}}+\beta_{k}^{n}\right)}{\frac{\phi_{k}^{n}}{\zeta^{n}}+\gamma_{k}^{n}-\beta_{k}^{n}}$

$D_{\mathrm{B}, k}^{n}=\frac{1}{2}\left[\left\{2 \hat{q}_{k}^{n}\left(\alpha_{k}^{n}\right)+2 \lambda_{\mathrm{A}, k}^{n}\left(\frac{\theta_{k}^{n}}{\zeta^{n}}+\beta_{k}^{n}-\alpha_{k}^{n}\right)+\mu_{\mathrm{B}, k}^{n}\left(\frac{\phi_{k}^{n}}{\zeta^{n}}+\gamma_{k}^{n}-\beta_{k}^{n}\right)\right\}\left(\frac{\phi_{k}^{n}}{\zeta^{n}}+\gamma_{k}^{n}-\beta_{k}^{n}\right)\right]$

The queue rate in lane $k$ for the effective green time in the $n$th cycle, $\mu_{\mathrm{B}, k}^{n}$, is shown as a formula to calculate a slope between $\left(\frac{\theta_{k}^{n}}{\zeta^{n}}+\beta_{k}^{n}, \hat{q}_{k}^{n}\left(\frac{\theta_{k}^{n}}{\zeta^{n}}+\beta_{k}^{n}\right)\right) \quad$ and $\left(\frac{\theta_{k}^{n}+\phi_{k}^{n}}{\zeta^{n}}+\gamma_{k}^{n}, \hat{q}_{k}^{n}\left(\frac{\theta_{k}^{n}+\phi_{k}^{n}}{\zeta^{n}}+\gamma_{k}^{n}\right)\right)$ in Equation (4). In Equation (5), the control delay in section B is calculated. The first term in a brace is the number of queued vehicles in lane $k$ at $\frac{\theta_{k}^{n}}{\zeta^{n}}+\beta_{k}^{n}$, $\hat{q}_{k}^{n}\left(\alpha_{k}^{n}\right)+\lambda_{\mathrm{A}, k}^{\mathrm{n}}\left(\frac{\theta_{k}^{n}}{\zeta^{n}}+\beta_{k}^{n}-\alpha_{k}^{n}\right)$, plus the queue lengths in lane $k$ at $\frac{\theta_{k}^{n}+\phi_{k}^{n}}{\zeta^{n}}+\gamma_{k}^{n}$, 
$\hat{q}_{k}^{n}\left(\alpha_{k}^{n}\right)+\lambda_{\mathrm{A}, k}^{\mathrm{n}}\left(\frac{\theta_{k}^{n}}{\zeta^{n}}+\beta_{k}^{n}-\alpha_{k}^{n}\right)+\mu_{\mathrm{B}, k}^{n}\left(\frac{\phi_{k}^{n}}{\zeta^{n}}+\gamma_{k}^{n}-\beta_{k}^{n}\right)$. The term in the second brace is a duration, $\frac{\phi_{k}^{n}}{\zeta^{n}}+\gamma_{k}^{n}-\beta_{k}^{n}$, in which the queue rate is negative in section B.

\subsubsection{Section $C$}

For section $\mathrm{C}$, we categorize queueing formation patterns into four forms of $\lambda_{\mathrm{C}, k}^{n}$ according to the traffic conditions described in Figure 5. If the queued vehicles in lane $k$ are still discharging during the red periods after the effective green time, $\gamma_{k}^{n}$ is set as the number of time units during which the queue rate is negative; otherwise, $\gamma_{k}^{n}$ is set as zero. The number of seconds during which no queued vehicles are present in lane $\mathrm{k}$ during the red time after the effective green time is shown as $\delta_{k}^{n}$ to adjust the start time of the arrival rate in section $\mathrm{C}$.

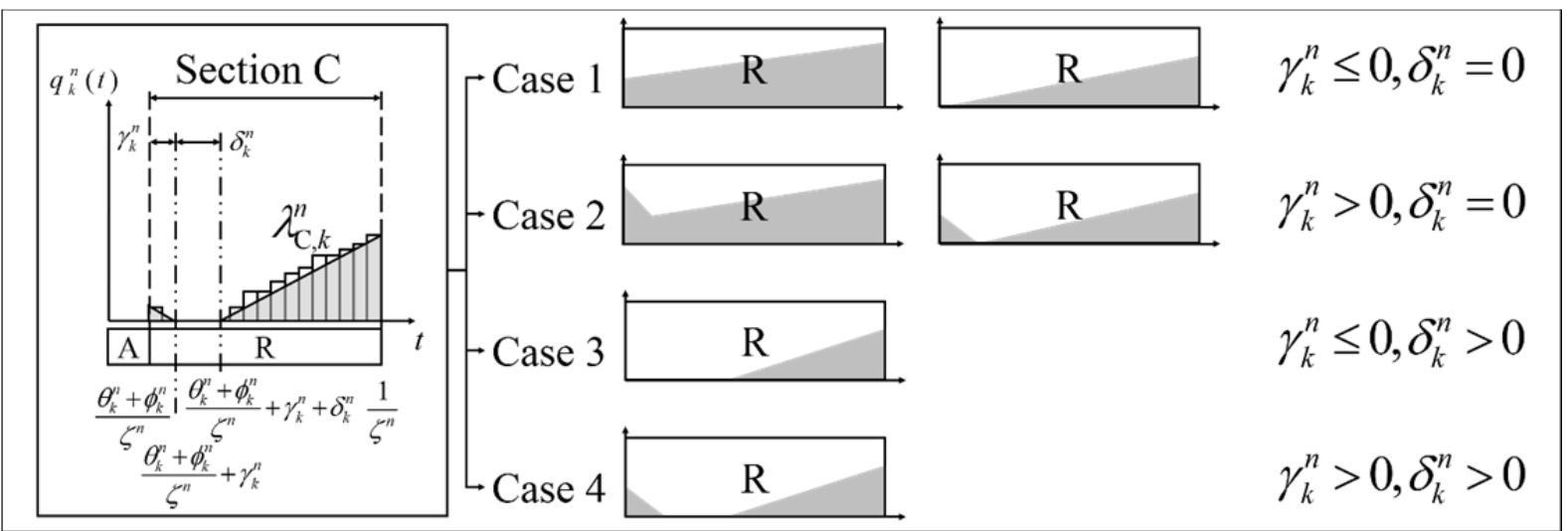

Figure 5. Four queueing formation patterns in Section C.

The formulations for the queue rate and the IQA are described in the following equations, including the adjustment factors, $\gamma_{k}^{n}$ and $\delta_{k}^{n}$.

$$
\begin{aligned}
\lambda_{\mathrm{C}, k}^{n}= & \frac{\hat{q}_{k}^{n}\left(\frac{1}{\zeta^{n}}\right)-\hat{q}_{k}^{n}\left(\frac{\theta_{k}^{n}+\phi_{k}^{n}}{\zeta^{n}}+\gamma_{k}^{n}+\delta_{k}^{n}\right)}{\frac{1}{\zeta^{n}}-\frac{\theta_{k}^{n}+\phi_{k}^{n}}{\zeta^{n}}-\gamma_{k}^{n}-\delta_{k}^{n}} \\
D_{\mathrm{C}, k}^{n} & =\frac{1}{2}\left[\left\{2 \hat{q}_{k}^{n}\left(\frac{1}{\zeta^{n}}\right)-\lambda_{\mathrm{C}, k}^{n}\left(\frac{1}{\zeta^{n}}-\frac{\theta_{k}^{n}+\phi_{k}^{n}}{\zeta^{n}}+\gamma_{k}^{n}+\delta_{k}^{n}\right)\right\}\left(\frac{1}{\zeta^{n}}-\frac{\theta_{k}^{n}+\phi_{k}^{n}}{\zeta^{n}}+\gamma_{k}^{n}+\delta_{k}^{n}\right)\right]
\end{aligned}
$$

The arrival rate in lane $k$ for the effective green time in the $n$th cycle, $\lambda_{\mathrm{C}, k}^{n}$, is shown as a formula to calculate a slope between $\left(\frac{\theta_{k}^{n}+\phi_{k}^{n}}{\zeta^{n}}+\gamma_{k}^{n}+\delta_{k}^{n}, \hat{q}_{k}^{n}\left(\frac{\theta_{k}^{n}+\phi_{k}^{n}}{\zeta^{n}}+\gamma_{k}^{n}+\delta_{k}^{n}\right)\right)$ and $\left(\frac{1}{\zeta^{n}}, \hat{q}_{k}^{n}\left(\frac{1}{\zeta^{n}}\right)\right)$ in Equation (6). In Equation (7), the IQA on section C is computed. The term in the 
first brace is the number of queued vehicles in lane $k$ at $\frac{\theta_{k}^{n}+\phi_{k}^{n}}{\zeta^{n}}+\gamma_{k}^{n}+\delta_{k}^{n}$, $\hat{q}_{k}^{n}\left(\frac{1}{\zeta^{n}}\right)-\lambda_{\mathrm{C}, k}^{n}\left(\frac{1}{\zeta^{n}}-\frac{\theta_{k}^{n}+\phi_{k}^{n}}{\zeta^{n}}+\gamma_{k}^{n}+\delta_{k}^{n}\right)$, plus the queue lengths in lane $k$ at $\frac{1}{\zeta^{n}}, \hat{q}_{k}^{n}\left(\frac{1}{\zeta^{n}}\right)$. The term in the second brace is a duration, $\frac{1}{\zeta^{n}}-\frac{\theta_{k}^{n}+\phi_{k}^{n}}{\zeta^{n}}+\gamma_{k}^{n}+\delta_{k}^{n}$, in which the arrival rate is negative in section $\mathrm{C}$.

\subsubsection{The total delay formula in the nth cycle}

For the total control delay formula in lane $k$, including all IQAs, the queueing formation patterns in this study are classified into 24 cases according to the combination of adjustment factors, rather than simple categorization into undersaturated or oversaturated traffic conditions. The queue formation patterns are described according to the adjustment factors in the following table.

Table 1 Queueing formation patterns according to the factors

\begin{tabular}{|c|c|l|}
\hline Factors & Sign & \\
\hline \multirow{2}{*}{$\alpha_{k}^{n}$} & $=0$ & It is highly possible that there is a residual queue. \\
\cline { 2 - 4 } & $>0$ & There is no residual queue. \\
\hline \multirow{2}{*}{$\beta_{k}^{n}$} & $=0$ & No vehicles are built up in the effective green time. \\
\cline { 2 - 3 } & $>0$ & Some vehicles are still built up in the effective green time. \\
\hline \multirow{3}{*}{$\gamma_{k}^{n}$} & $<0$ & All vehicles are discharged within the effective green time. \\
\cline { 2 - 3 } & $=0$ & It is possible that all vehicles are not discharged within the effective green time \\
\cline { 2 - 3 } & $>0$ & $\begin{array}{l}\text { Some vehicles are still discharging in the red time, and it is highly possible that } \\
\text { queued vehicles remain. }\end{array}$ \\
\hline \multirow{2}{*}{$\delta_{k}^{n}$} & $=0$ & It is possible that there is heavy traffic flow after the effective green time. There \\
\cline { 2 - 3 } & $>0$ & is no heavy traffic flow after the effective green time. \\
\hline
\end{tabular}

$\alpha_{k}^{n}$ shows the existence of a residual queue at the start time of a cycle. If there is a residual queue, $\alpha_{k}^{n}$ is definitely set as zero. In addition, if the vehicles are arrived from the start of a cycle, despite the absence of a residual queue, $\alpha_{k}^{n}$ can be also set as zero. The meaning of the value of $\beta_{k}^{n}$ is relatively simple. If the number of queued vehicle is still increasing during the effective green time, $\beta_{k}^{n}$ is set as the number of seconds, in which the arrival rate is a positive; otherwise, it is set as zero. $\gamma_{k}^{n}$ describes whether the current traffic condition is oversaturated or undersaturated regardless of the residual queue at the start of a cycle. If all vehicles are left from lane $k$ during the effective green time, $\gamma_{k}^{n}$ is set as the number of seconds during which lane $k$ is empty, with a negative sign. In contrast, if the vehicle discharge process is finished at the end of the effective green time in both cases in which the queued vehicles remain or do not remain, $\gamma_{k}^{n}$ is set as zero. If the queues are still discharging after the effective green time in both of the aforementioned cases, $\gamma_{k}^{n}$ is set as the number of seconds during which the queue rate is a positive in the red time. $\delta_{k}^{n}$ shows whether queued vehicles remain. If it is highly possible that there is a residual queue and heavy traffic flow after the effective green time, $\delta_{k}^{n}$ is set as zero; otherwise, it is set as the number of seconds during which lane $k$ is empty. According to the value and the sign of the factors, the mathematical formulations are applied differently for each lane on each cycle. The basic form of the control delay in the $n$th cycle is given in Equation (8). 


$$
D^{n}=\frac{1}{2} \sum_{k=1}^{K}\left[\begin{array}{l}
\left\{2 \hat{q}_{k}^{n}\left(\alpha_{k}^{n}\right)+\lambda_{\mathrm{A}, k}^{n}\left(\frac{\theta_{k}^{n}}{\zeta^{n}}+\beta_{k}^{n}-\alpha_{k}^{n}\right)\right\}\left(\frac{\theta_{k}^{n}}{\zeta^{n}}+\beta_{k}^{n}-\alpha_{k}^{n}\right) \\
+\left\{2 \hat{q}_{k}^{n}\left(\alpha_{k}^{n}\right)+2 \lambda_{\mathrm{A}, k}^{n}\left(\frac{\theta_{k}^{n}}{\zeta^{n}}+\beta_{k}^{n}-\alpha_{k}^{n}\right)+\mu_{\mathrm{B}, k}^{n}\left(\frac{\phi_{k}^{n}}{\zeta^{n}}+\gamma_{k}^{n}-\beta_{k}^{n}\right)\right\}\left(\frac{\phi_{k}^{n}}{\zeta^{n}}+\gamma_{k}^{n}-\beta_{k}^{n}\right) \\
+\left\{2 \hat{q}_{k}^{n}\left(\frac{1}{\zeta^{n}}\right)-\lambda_{\mathrm{C}, k}^{n}\left(\frac{1}{\zeta^{n}}-\frac{\theta_{k}^{n}+\phi_{k}^{n}}{\zeta^{n}}+\gamma_{k}^{n}+\delta_{k}^{n}\right)\right\}\left(\frac{1}{\zeta^{n}}-\frac{\theta_{k}^{n}+\phi_{k}^{n}}{\zeta^{n}}+\gamma_{k}^{n}+\delta_{k}^{n}\right)
\end{array}\right]
$$

To prevent the abnormal predicted values of components during rolling horizon periods, Equation (8) is transformed on the basis of four adjustment factors that describe the traffic conditions during the $n$th cycle. Consequently, the formulation of the control delay is organized as the following equation and sets of variables.

$$
\begin{aligned}
& D^{n}=\frac{1}{2} \sum_{k=1}^{K}\left[D_{\mathrm{A}, k}^{n}\left(\Gamma^{n}, \boldsymbol{\Omega}_{k}^{n}, \boldsymbol{\Phi}_{k}^{n}\right)+D_{\mathrm{B}, k}^{n}\left(\Gamma^{n}, \boldsymbol{\Omega}_{k}^{n}, \boldsymbol{\Phi}_{k}^{n}\right)+D_{\mathrm{B}, k}^{n}\left(\Gamma^{n}, \boldsymbol{\Omega}_{k}^{n}, \boldsymbol{\Phi}_{k}^{n}\right)\right] \\
& \Gamma^{n}=\left\{\zeta^{n}, \theta_{1}^{n} \ldots \theta_{k}^{n}, \phi_{1}^{n} \ldots \theta_{k}^{n}\right\}, \forall k \in K, \forall n \in N \\
& \boldsymbol{\Omega}_{k}^{n}=\left\{\alpha_{k}^{n}, \beta_{k}^{n}, \gamma_{k}^{n}, \delta_{k}^{n}\right\}, \forall k \in K, \forall n \in N \\
& \boldsymbol{\Psi}_{k}^{n}=\left\{\hat{q}_{k}^{n}\left(\alpha_{k}^{n}\right), \hat{q}_{k}^{n}\left(\frac{\theta_{k}^{n}}{\zeta^{n}}+\beta_{k}^{n}\right), \hat{q}_{k}^{n}\left(\frac{\theta_{k}^{n}+\phi_{k}^{n}}{\zeta^{n}}+\gamma_{k}^{n}\right), \hat{q}_{k}^{n}\left(\frac{\theta_{k}^{n}+\phi_{k}^{n}}{\zeta^{n}}+\gamma_{k}^{n}+\delta_{k}^{n}\right), \hat{q}_{k}^{n}\left(\frac{1}{\zeta^{n}}\right)\right\}, \forall k \in K, \forall n \in N \\
& \boldsymbol{\Phi}_{k}^{n}=\left\{\lambda_{\mathrm{A}, k}^{n}, \mu_{\mathrm{B}, k}^{n}, \lambda_{\mathrm{C}, k}^{n}\right\}, \forall k \in K, \forall n \in N
\end{aligned}
$$

Equation (9), with four sets of components, is identical to Equation (8). $\Gamma^{n}$ is the set of group-based variables, which is optimized on the basis of the predicted parameters in the optimization process for future study. $\boldsymbol{\Omega}_{k}^{n}$ is the set of adjustment factors used to decide the current states of queueing formation patterns in the current cycle. $\Psi_{k}^{n}$ is the set of queue lengths at an inflection point. $\Omega_{k}^{n}$ and $\Psi_{k}^{n}$ are used as seed parameters to predict future traffic patterns in a rolling horizon procedure. $\Phi_{k}^{n}$ is the set of slopes, including the arrival rates before an effective green time, discharge rates, and the arrival rates after an effective green time. $\Phi_{k}^{n}$ is computed on the basis of the predicted $\boldsymbol{\Omega}_{k}^{n}$ and $\Psi_{k}^{n}$ on a rolling horizon step. The computed $\boldsymbol{\Phi}_{k}^{n}$ and the predicted $\boldsymbol{\Omega}_{k}^{n}$ are then used to estimate the future control delay in appropriate time windows with optimized $\Gamma^{n}$.

\subsection{Lane-based delay predictive model based on Kalman filters}

We use the Kalman filter, which is an effective and efficient recursive process for time series analysis introduced by Welch (2001), in signal processing. To start the rolling horizon procedure as a recursive process in this research, we set the system variables, time update equations, and measurement equations. The set of adjustment factors, $\boldsymbol{\Omega}_{k}^{n-1}$, and the set of queue lengths at an inflection point, $\Psi_{k}^{n-1}$, in the (n-1)th cycle are defined as the measurement variables, because the recursive procedures and the optimization procedures are performed in the current cycle, i.e., the $n$th cycle. For the future time windows, $\boldsymbol{\Omega}_{k}^{n+x}$ and $\Psi_{k}^{n+x}$ are defined as the state variables for cycle $n$ with a variable $x$, which is the future cycle from the current $n$th cycle. The rolling horizon process of the Kalman filter in estimating $\hat{\mathbf{\Omega}}_{k}^{n+x}$ and $\hat{\mathbf{\Psi}}_{k}^{n+x}$ is described in Table 2, which is the abridged version 
of the process in Lee et al. (2015b). $\hat{\mathbf{\Omega}}_{k}^{n+x}$ and $\hat{\mathbf{\Psi}}_{k}^{n+x}$ are then used to estimate $\hat{\boldsymbol{\Phi}}_{k}^{n+x}$ using equations (2), (4), and (6). $\hat{\Omega}_{k}^{n+x}, \hat{\Psi}_{k}^{n+x}$, and $\hat{\Phi}_{k}^{n+1}$ are then used to estimate the total control delay in the $(n+x)$ th cycle, $D^{n+x}$ using Equation (9). A superscript (-) indicates an a priori state estimate at step $i$, and a circumflex $(\wedge)$ indicates an a posteriori state estimate at cycle $n$. The step of the recursive process is set as $i$.

Table 2. Rolling horizon procedure based on Kalman filter for all time windows.

\begin{tabular}{|c|c|}
\hline- & Equations \\
\hline \multirow{3}{*}{$\begin{array}{l}\text { Time Updat } \\
\text { Equations }\end{array}$} & 1) Initial estimates for $\left(\hat{\Omega}_{k}^{n+x}\right)^{-1(-)},\left(P_{\Omega, k}\right)^{i-1(-)},\left(\hat{\Psi}_{k}^{n+x}\right)^{i-1(-)}$, and $\left(P_{\Psi, k}\right)^{i-1(-)}$ \\
\hline & $\begin{array}{l}\text { 2) Project the state from }(i-1) \text { to } i \\
\left(\boldsymbol{\Omega}_{k}^{n+x}\right)^{i}=A_{\Omega, k}\left(\boldsymbol{\Omega}_{k}^{n+x}\right)^{i-1}+w_{\boldsymbol{\Omega}, k}, P\left(w_{\boldsymbol{\Omega}, k}\right) \sim N\left(0, Q_{\Omega, k}\right) \\
\left(\boldsymbol{\Psi}_{k}^{n+x}\right)^{i}=A_{\Psi, k}\left(\Psi_{k}^{n+x}\right)^{i-1}+w_{\Psi, k}, P\left(w_{\Psi, k}\right) \sim N\left(0, Q_{\Psi, k}\right) \\
\left(\hat{\boldsymbol{\Omega}}_{k}^{n+x}\right)^{i(-)}=A_{\Omega, k}\left(\hat{\boldsymbol{\Omega}}_{k}^{n+x}\right)^{i-1(-)}+w_{\Omega, k},\left(\hat{\Psi}_{k}^{n+x}\right)^{i(-)}=A_{\Psi, k}\left(\hat{\Psi}_{k}^{n+x}\right)^{i-1(-)}+w_{\Psi, k}\end{array}$ \\
\hline & $\begin{array}{l}\text { 3) Project the covariance estimates from }(i-1) \text { to } i \\
\text { the a priori estimate error covariance is } \\
P_{\Omega, k}^{n+x(-)}=E\left[e_{\Omega, k}^{n+x(-)} e_{\Omega, k}^{n+x(-)^{T}}\right], P_{\Psi, k}^{n+x(-)}=E\left[e_{\Psi, k}^{n+x(-)} e_{\Psi, k}^{n+x(-)^{T}}\right] \\
\text { the a posteriori estimate error covariance is } \\
P_{\Omega, k}^{n+x}=E\left[e_{\Omega, k}^{n+x} e_{\Omega, k}^{n+x^{T}}\right], P_{\Psi, k}^{n+x}=E\left[e_{\Psi, k}^{n+x} e_{\Psi, k}^{n+x^{T}}\right] \\
\left(P_{\Omega, k}^{n+x}\right)^{i(-)}=A_{\Omega, k}\left(P_{\Omega, k}^{n+x}\right)^{i(-)} A_{\Omega, k}{ }^{T}+Q_{\Omega, k},\left(P_{\Psi, k}^{n+x}\right)^{i(-)}=A_{\Psi, k}\left(P_{\Psi, k}^{n+x}\right)^{i(-)} A_{\Psi, k}{ }^{T}+Q_{\Psi, k}\end{array}$ \\
\hline \multirow{3}{*}{$\begin{array}{l}\text { Measurement } \\
\text { Equations }\end{array}$} & $\begin{array}{l}\text { 1) Compute the Kalman gain } \\
\boldsymbol{\Omega}_{k}^{n-1} \equiv H_{\Omega, k} \boldsymbol{\Omega}_{k}^{n+x}+v_{\Omega, k}, P\left(v_{\Omega, k}\right) \sim N\left(0, R_{\Omega, k}\right) \\
\Psi_{k}^{n-1} \equiv H_{\Psi, k} \Psi_{k}^{n+x}+v_{\Psi, k}, P\left(v_{\Psi, k}\right) \sim N\left(0, R_{\Psi, k}\right) \\
\left(K_{\Omega, k}^{n+x}\right)^{i}=\left(P_{\Omega, k}^{n+x}\right)^{i(-)} H_{\Omega, k}{ }^{T}\left\{H_{\Omega, k}\left(P_{\Omega, k}^{n+x}\right)^{i(-)} H_{\Omega, k}{ }^{T}+R_{\Omega, k}\right\}^{-1} \\
\left(K_{\Psi, k}^{n+x}\right)^{i}=\left(P_{\Psi, k}^{n+x}\right)^{i(-)} H_{\Psi, k}{ }^{T}\left\{H_{\Psi, k}\left(P_{\Psi, k}^{n+x}\right)^{i(-)} H_{\Psi, k}{ }^{T}+R_{\Psi, k}\right\}^{-1}\end{array}$ \\
\hline & $\begin{array}{l}\text { 2) Generate } \text { a posteriori state estimate by incorporating the measurement } \\
\left.\left(\hat{\boldsymbol{\Omega}}_{k}^{n+x}\right)^{i}=\left(\hat{\boldsymbol{\Omega}}_{k}^{n+x}\right)^{i(-)}+\left(K_{\Omega, k}^{n+x}\right)^{i} \boldsymbol{\Omega}_{k}^{n-1}-H_{\Omega, k}\left(\hat{\boldsymbol{\Omega}}_{k}^{n+x}\right)^{i(-)}\right\} \\
\left(\hat{\Psi}_{k}^{n+x}\right)^{i}=\left(\hat{\Psi}_{k}^{n+x}\right)^{i(-)}+\left(K_{\Psi, k}^{n+x}\right)^{i}\left\{\Psi_{k}^{n-1}-H_{\Psi, k}\left(\hat{\Psi}_{k}^{n+x}\right)^{i(-)}\right\}\end{array}$ \\
\hline & $\begin{array}{l}\text { 2) Update the error covariance } \\
\left(P_{\Omega, k}^{n+x}\right)^{i}=\left\{I-\left(K_{\Omega, k}^{n+x}\right)^{i} H_{\Omega, k}\right\}\left(P_{\Omega, k}^{n+x}\right)^{i(-)},\left(P_{\Psi, k}^{n+x}\right)^{i}=\left\{I-\left(K_{\Psi, k}^{n+x}\right)^{i} H_{\Psi, k}\right\}\left(P_{\Psi, k}^{n+x}\right)^{i(-)}\end{array}$ \\
\hline
\end{tabular}

The system parameters, $\mathbf{P}=\left\{A_{\Omega, k}, Q_{\Omega, k}, H_{\Omega, k}, R_{\Omega, k}, A_{\Psi, k}, Q_{\Psi, k}, H_{\Psi, k}, R_{\Psi, k}\right\}, \forall k \in K$, are calibrated in each future cycle, $x=x 1, x 2, x 3, \ldots$, according to the set of adjustment factors and the set of queue lengths at an inflection point. The concept of rolling horizon procedures, data processing, and the most appropriate time windows are illustrated in Figure 6. 


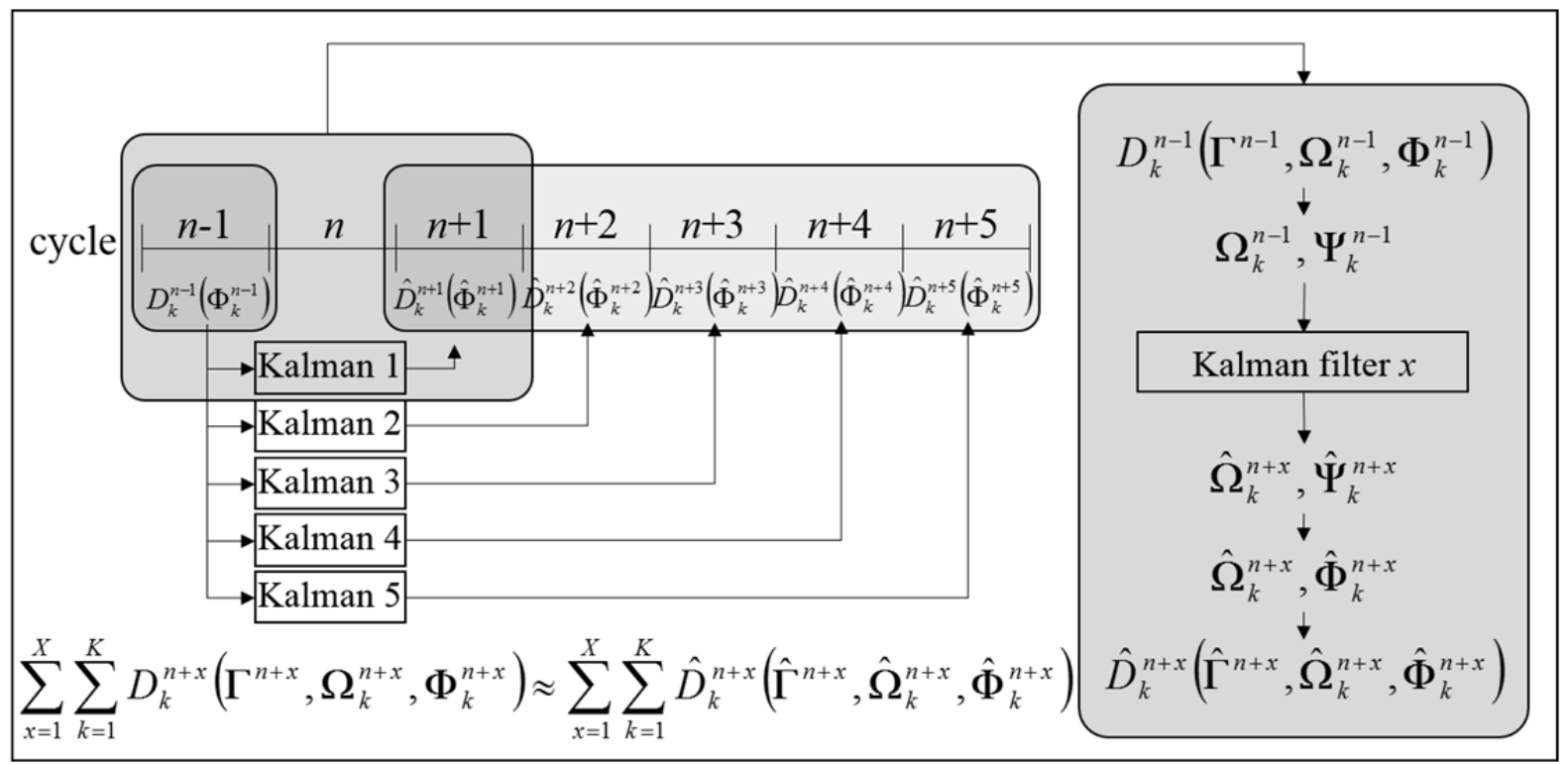

Figure 6 . The concept of a rolling horizon procedure.

In Figure $6, \Omega_{k}^{n-1}$ and $\Psi_{k}^{n-1}$ are extracted as the seed data for the recursive procedure, i.e., Kalman filtering. These data are used in the Kalman filter as the measurement variables and then, the future $\boldsymbol{\Omega}_{k}^{n+x}$ and $\Psi_{k}^{n+x}$ are predicted as $\hat{\boldsymbol{\Omega}}_{k}^{n+x}$ and $\hat{\mathbf{\Psi}}_{k}^{n+x}$ to calculate $\hat{\boldsymbol{\Phi}}_{k}^{n+x}$. The control delay in lane $k$ in the $(n+x)$ th cycle is computed based on $\hat{\Omega}_{k}^{n+x}$ and $\hat{\Phi}_{k}^{n+x}$ with optimized group-based variables, $\hat{\Gamma}_{k}^{n+x} . X$ is the most appropriate time window to maintain the robustness and the accuracy of a predictive procedures to show the smallest error between the observed control delay and the predicted control delay at the calibration process.

\section{CALIBRATION PROCEDURES}

We used a typical directional three-lane, four-arm approach at an isolated intersection to calibrate the proposed method using VISSIM computer software. The VISSIM COM (Component Objective Model) interface was used to install an overall process: collection of data, estimation of variables, calculation of parameters, and rolling horizon procedures, with visual basic for applications). The vehicle-actuated programming in VISSIM was used to generate the impulse memories and time occupancy rates of the upstream and downstream detectors.

A four-phase signalized intersection with a cycle length of $60 \mathrm{~s}$ was created. The green signal duration for each phase was $10 \mathrm{~s}$, with an inter-green time of $5 \mathrm{~s}$. A four-phase signalized intersection with control algorithms was created based on the COM interface. A full set of $2 \times 2-\mathrm{m}$ loop detectors was installed to record the impulse memory and time occupancy rate data. The upstream detectors were positioned $200 \mathrm{~m}$ from the stop-line. Left turns on red were prohibited, in accordance with the driving rule for left-hand traffic. The geometric layout of the simulated intersection is presented in Figure 7. 


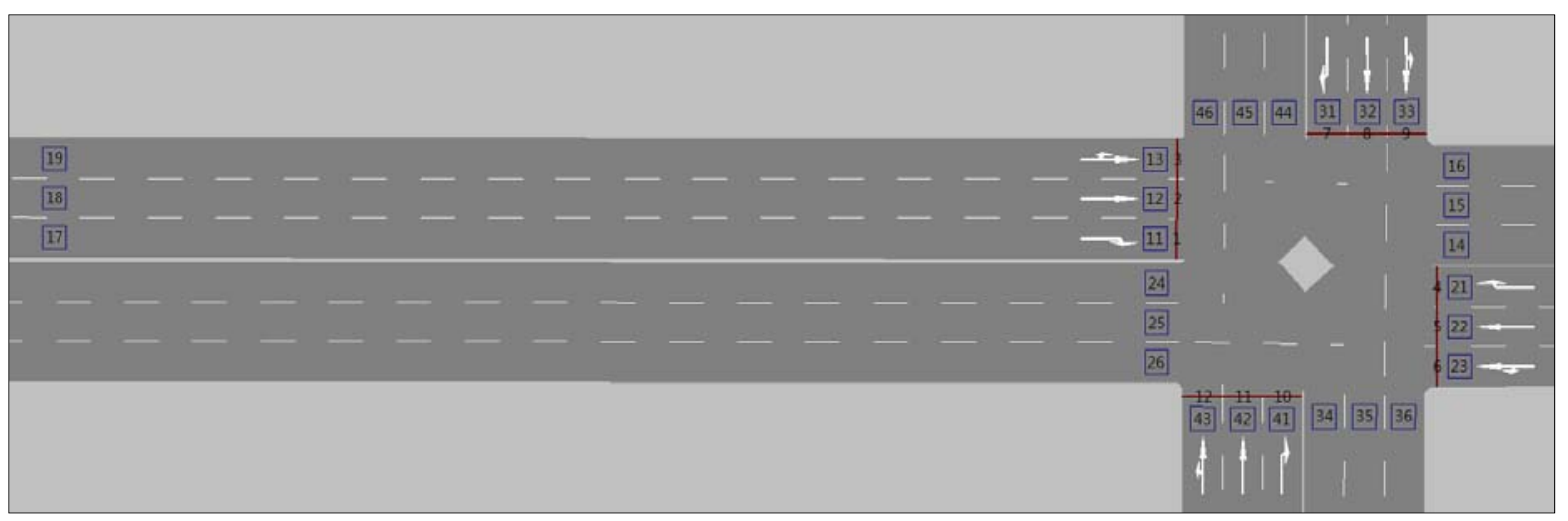

Figure 7. Geometric layout of simulated junction in VISSIM.

The simulation period was $18,000 \mathrm{~s}$, the simulation resolution was $1 \mathrm{~s}$, and the first and last $600 \mathrm{~s}$ were used as warm-up and cool-down periods. We further assumed that the turning proportions were evenly distributed among all of the movements. One third of the vehicles were assigned to each turning movement for data calibration. The stage structure is eastbound, westbound, southbound, and northbound. Each stage has three traffic streams; left-turn, right-turn, and passing-through traffic. Seven levels of traffic volume (degrees of saturation), i.e., $700(0.8), 750$ (0.86), 800 (0.92), 850 (0.97), 900 (1.03), 950 (1.09), and 1000 (1.14) vph, were set for all of the approaches for each 2000-s interval for model calibration and validation. To calibrate the system parameters on the Kalman filter, we used the estimated lane-based queue lengths in the aforementioned signalized junction in 12 lanes in every 290 cycles.

For the lane-based IQA based on group-based variables, the estimates of the proposed IQA method and the IQA method without adjustment factors were compared in this study. In each method, the estimated IQAs are presented with the values of control delay, which includes lane-based estimated queue lengths, in 3480 cases (12 lanes $\times 290$ cycles) in the following figure.

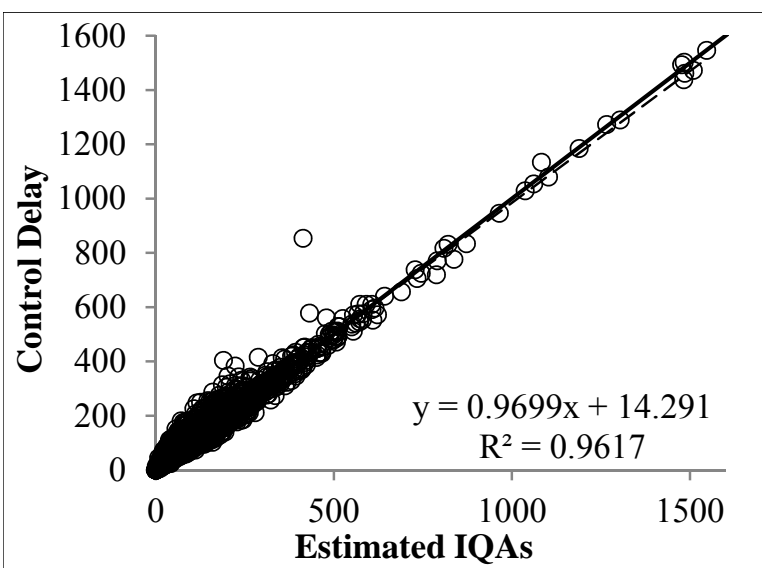

(a) Proposed IQA without adjustment factors.

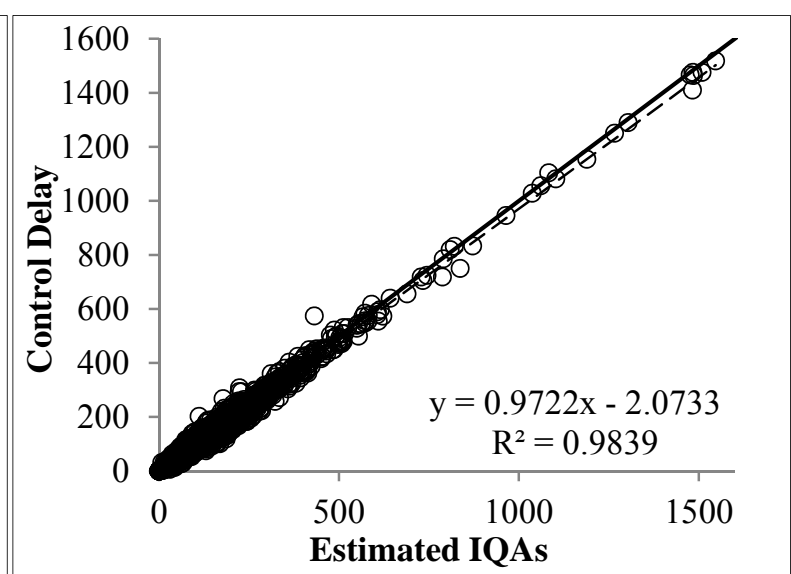

(b) Proposed IQA with adjustment factors.

Figure 8. Scatter plot of estimates of IQA on calibration set.

In Figure 8, both graphs demonstrate that the proposed lane-based IQA method estimates well the control delay in each lane in each cycle. For the proposed IQA method without adjustment factors, even if the estimates of IQAs are distributed around the diagonal line with a high $\mathrm{R}^{2}$ value, 0.9617 , the estimates are not narrowly scattered around the line with a relatively higher constant, $14.291 \mathrm{veh} \cdot \mathrm{s}$, especially under low-traffic volume conditions. The values of root mean square error (RMSE) and mean absolute percent error (MAPE) are $25.76 \mathrm{veh} \cdot \mathrm{s}$ and $22.23 \%$, respectively. For the proposed IQA method with adjustment factors, the estimated IQAs are densely scattered on the periphery of the diagonal line, the $\mathrm{R}^{2}$ value is 0.9837 , and the coefficient, 0.9722 , is close to one. The low constant, $-2.0733 \mathrm{veh} \cdot \mathrm{s}$, shows that the estimates are almost identical with the control delay. In addition, the 
values of RMSE and MAPE are $16.5 \mathrm{veh} \cdot \mathrm{s}$ and $12.8 \%$, respectively, for the proposed models, which are $9.26 \mathrm{veh} \cdot \mathrm{s}$ and $9.43 \%$, respectively, lower than the values of the method that does not include adjustment factors. According to the RMSE and MAPE values and the distribution of the estimates, the proposed IQA estimation method performs very well under conditions of low and high traffic flow.

For the rolling horizon procedures, we calibrated the system parameters in the Kalman filter. The total number of system parameters is 2400 , because there are 4 parameters $(A, Q, H, R), 5$ cycles $(x)$ as the future time windows, 12 lanes $(k)$, and 10 estimates $\left(\hat{\mathbf{\Omega}}_{k}^{n+x}\right.$ and $\left.\hat{\mathbf{\Psi}}_{k}^{n+x}\right)$. The Kalman system parameters describes the relationship adjustment factors and queue lengths at an inflection point between the $(n-1)$ th cycle and the $(n+x)$ th cycle within the future time windows. After calibration, the Kalman system parameters were used in the specified geographic conditions regardless of the traffic conditions and signal settings. The calibration results are specified in Appendix I. If there was a zero parameter after calibration, that parameter was set as 0.00001 because the recursive process malfunctions when one of the system parameters is set as zero, at least.

\section{VALIDATION RESULTS}

We used the lane-based observed queue lengths directly extracted from the trajectories in VISSIM to validate the effectiveness of the proposed IQA method and the rolling horizon procedure. To demonstrate the distinctive strength of the developed methods, a comparison of the various estimation methods of lane-based control delay prediction based on various model structures is presented in this section. The specifications of the proposed models are summarized in Table 3.

Table 3. Descriptions of the proposed models.

\begin{tabular}{|c|c|c|}
\hline Model & IQAs & Rolling horizon process \\
\hline $1^{*}$ & $\begin{array}{l}\text { Developed estimation method for IQAs } \\
\text { without adjustment factors }\end{array}$ & $\begin{array}{l}\text { We suppose that the components of control } \\
\text { delay in the future time windows are simply } \\
\text { the same as those in the }(n-1) \text { th cycle. }\end{array}$ \\
\hline 2 & $\begin{array}{l}\text { Developed estimation method for IQAs } \\
\text { without adjustment factors }\end{array}$ & $\begin{array}{l}\text { We suppose that the components of control } \\
\text { delay in the future time windows except the } \\
(n+1) \text { th cycle follow the predicted } \\
\text { components of control delay in the }(n+1) \text { th } \\
\text { cycle using the Kalman filters. }\end{array}$ \\
\hline 3 & $\begin{array}{l}\text { Developed estimation method for IQAs } \\
\text { without adjustment factors }\end{array}$ & $\begin{array}{l}\text { Components of control delay in all future } \\
\text { time windows are predicted on the basis of } \\
\text { those in the }(n-1) \text { th cycle using the Kalman } \\
\text { filters. }\end{array}$ \\
\hline 4 & $\begin{array}{l}\text { Developed estimation method for IQAs with } \\
\text { adjustment factors }\end{array}$ & $\begin{array}{l}\text { We suppose that the components of control } \\
\text { delay in the future time windows are simply } \\
\text { the same as those in the }(n-1) \text { th cycle. }\end{array}$ \\
\hline 5 & $\begin{array}{l}\text { Developed estimation method for IQAs with } \\
\text { adjustment factors }\end{array}$ & $\begin{array}{l}\text { We suppose that the components of control } \\
\text { delay in the future time windows except the } \\
(n+1) \text { th cycle follow the predicted } \\
\text { components of control delay in the }(n+1) \text { th } \\
\text { cycle using the Kalman filters. }\end{array}$ \\
\hline 6 & $\begin{array}{l}\text { Developed estimation method for IQAs with } \\
\text { adjustment factors }\end{array}$ & $\begin{array}{l}\text { Components of control delay in all future } \\
\text { time windows are predicted on the basis of } \\
\text { those in the }(n-1) \text { th cycle using the Kalman } \\
\text { filters. }\end{array}$ \\
\hline
\end{tabular}

*Reference model: basic form of proposed method without detailed processing.

In Table 3, the most widely used average delay formula, the Webster's delay equation, is not set as the reference model. This choice is made for two reasons. First, the analysis time for these cases 
is 60 seconds, which is not suitable for the estimation of Webster's delay in each cycle. This is because the Webster's delay equation represents the steady-state traffic conditions for a relatively long analysis period. The Webster's delay equation consists of a uniform component, a random component, and a statistical fitting to estimate the average delay, based on accumulated arrivals and departures during a reasonable period of several cycles. The equation is therefore only suitable for estimating the average delay in steady-state conditions in the long run. In this study, the values of traffic intensities for each cycle (which are based on the traffic information collected during a cycle of about 60 seconds) are short-term quantities that describe a particular incident of a statistical distribution, which can substantially deviate from the averaging conditions modeled by the Webster's delay equation. Second, Webster's delay equation is not fitted to estimate the lane-based control delay cycle-by-cycle, due to the lack of temporal and spatial information on queue lengths second-bysecond at the micro-level. This is because we cannot consider lane-changing behavior for lane-based queue lengths or the transient fluctuations of traffic flows at micro levels, second-by-second and cycle-by-cycle, by using this equation. The average traffic intensities, the effective green times, and the cycle times are only considered in the Webster's delay equation for approximating the average delay. Consequently, the Webster's delay equation is not appropriate for estimating the real-time control delay for each lane in each cycle, even though this equation shows excellent performance under steady-state, under-saturated traffic conditions.

In this study, model 1 follows the general equation of the IQA estimation methods proposed in HCM 2010, which is used as the reference model. Even if only the concept of the lane-based IQA estimation method is provided in HCM 2010 (without a specific calculation procedure, because of the absence of lane-based temporal and spatial information under general conditions), we can develop the specific calculation procedure on the basis of the estimated lane-based queue lengths. In model 1, the rolling horizon procedure is also a fundamental form, by which the traffic conditions in the near future cycle simply follow those of the recent past cycle. The lane-based IQA estimation methods used in models 2 and 3 are identical to those used in model 1 . However, the rolling horizon procedures are applied differently in these models. In model 2, only the components of control delay in the nearest future cycle, i.e., the $(n+1)$ th cycle, are predicted with the Kalman recursive process, and the control delay in the remaining future cycles in the time windows simply follows the components predicted in the $(n+1)$ th cycle. However, the components of control delay in all future cycles in the time windows are separately predicted by using their own Kalman recursive processes. For models 4, 5, and 6, we use the adjustment factors in the proposed estimation method for lane-based IQAs to improve the robustness and accuracy of the proposed model. Rolling horizon methods are then applied for models 4, 5, and 6 in an identical manner as they are applied in models 1, 2, and 3, respectively Accordingly, model 6 is the most developed form of the methods proposed in both the IQAs and in recursive processing.

The scatter plots of IQAs with or without factors for the proposed estimation method of lanebased IQAs are illustrated in Figure 9. In each scatter plot, the estimated IQAs are presented with the values of control delay, which include lane-based estimated queue lengths, in 3480 cases (12 lanes $\times$ 290 cycles). 


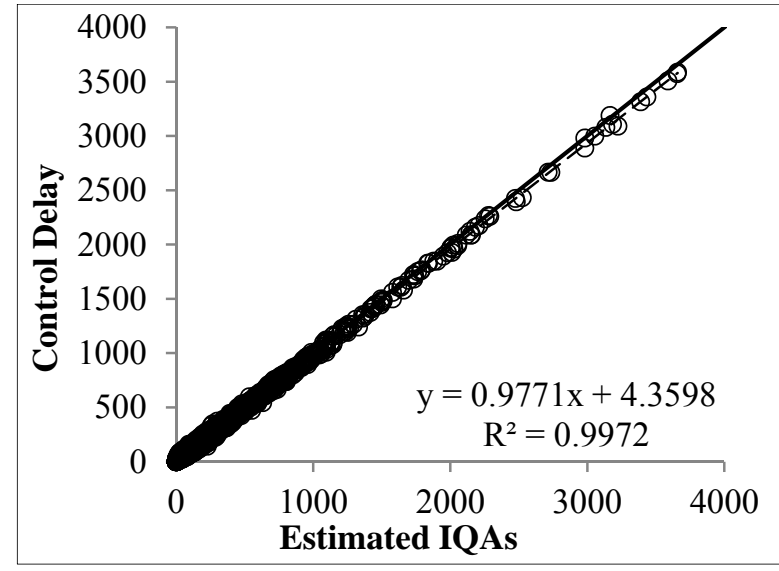

(a) Proposed IQA without adjustment factors.

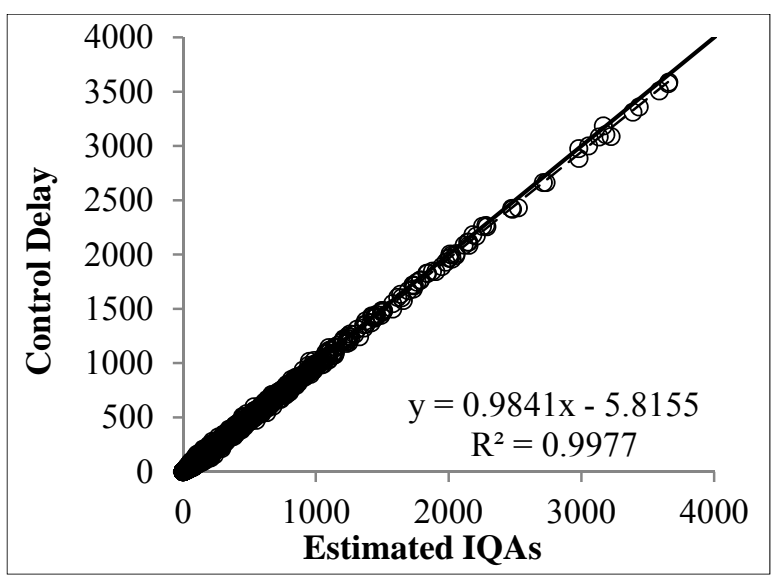

(b) Proposed IQA with adjustment factors.

Figure 9. Scatter plot of estimates of IQA on validation set.

In Figure 9, the proposed IQA estimation method, both without and with adjustment factors, clearly performs well for estimation of the control delay with high $\mathrm{R}^{2}$ values $(0.9972$ and 0.9977 , respectively). The RMSE values of the two methods are 19.79 and $19.74 \mathrm{veh} \cdot \mathrm{s}$, respectively, and the MAPE values are $16.62 \%$ and $11.33 \%$, respectively. According to the factors of model evaluations, which do not differ significantly from the factors in the calibration set, the proposed IQA estimation methods are effective and robust under diverse traffic conditions regardless of the use of adjustment factors.

For the rolling horizon procedure, we assumed maximum time windows of 5 minutes and 5 cycles to guarantee the relatively continuity and consistency of the queue formation patterns in this study. This means that the variable $X$ is set as 5 cycles in the following equation, which is shown to be the comparison of the estimated control delay using the recursive process to the observed queued control delay.

$$
\sum_{x=1}^{X} \sum_{k=1}^{K} D_{k}^{n+x}\left(\Gamma^{n+x}, \boldsymbol{\Omega}_{k}^{n+x}, \boldsymbol{\Phi}_{k}^{n+x}\right) \approx \sum_{x=1}^{X} \sum_{k=1}^{K} \hat{D}_{k}^{n+x}\left(\hat{\Gamma}^{n+x}, \hat{\mathbf{\Omega}}_{k}^{n+x}, \hat{\boldsymbol{\Phi}}_{k}^{n+x}\right)
$$

We examined the scatter plots of the aforementioned six models under the maximum time window condition and then chose two models based on the result on the previous steps. We compared two models according to the number of time windows based on the values of $\mathrm{R}^{2}$, RMSE, and MAPE to determine the most appropriate time windows and the most effective and robust predictive model. The scatter plots of the six proposed models are illustrated in Figure 10.

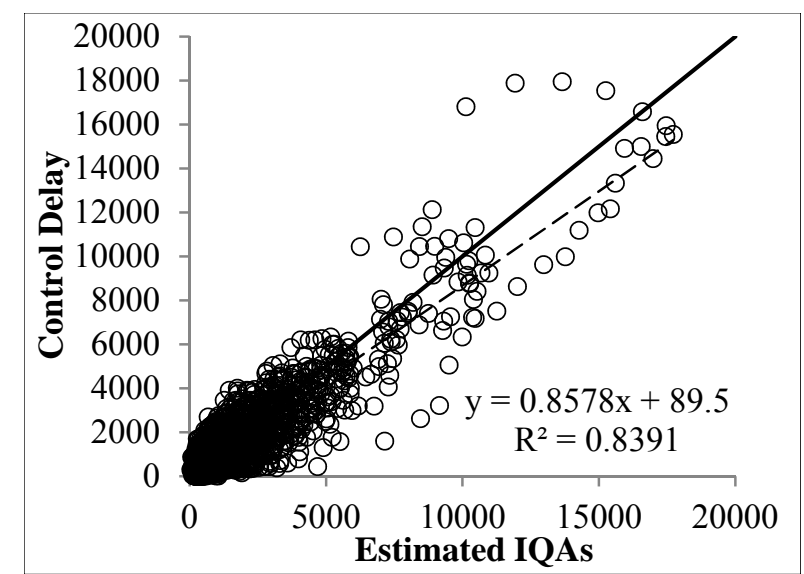

Model 1 (RMSE: $704.04 \mathrm{veh} \cdot \mathrm{sec}, \mathrm{MAPE}: 50.28 \%$ )

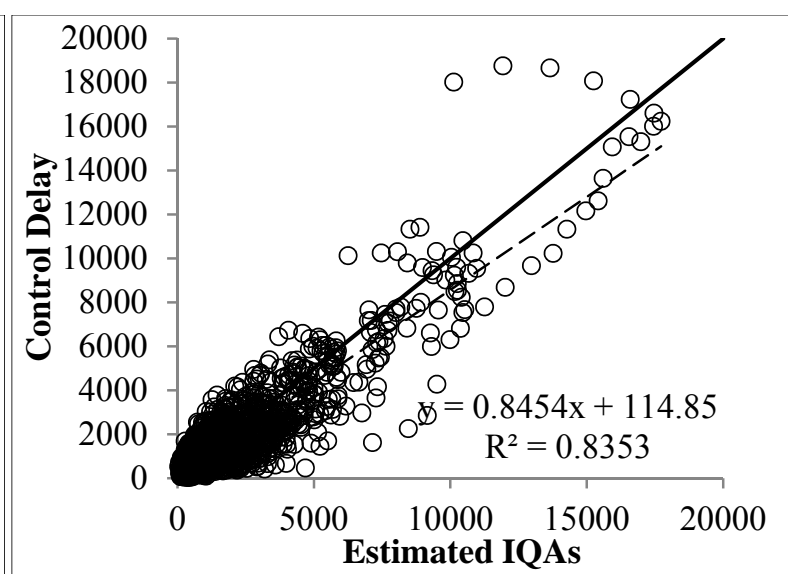

Model 2 (RMSE: $710.50 \mathrm{veh} \cdot \mathrm{sec}, \mathrm{MAPE}: 47.55 \%$ ) 


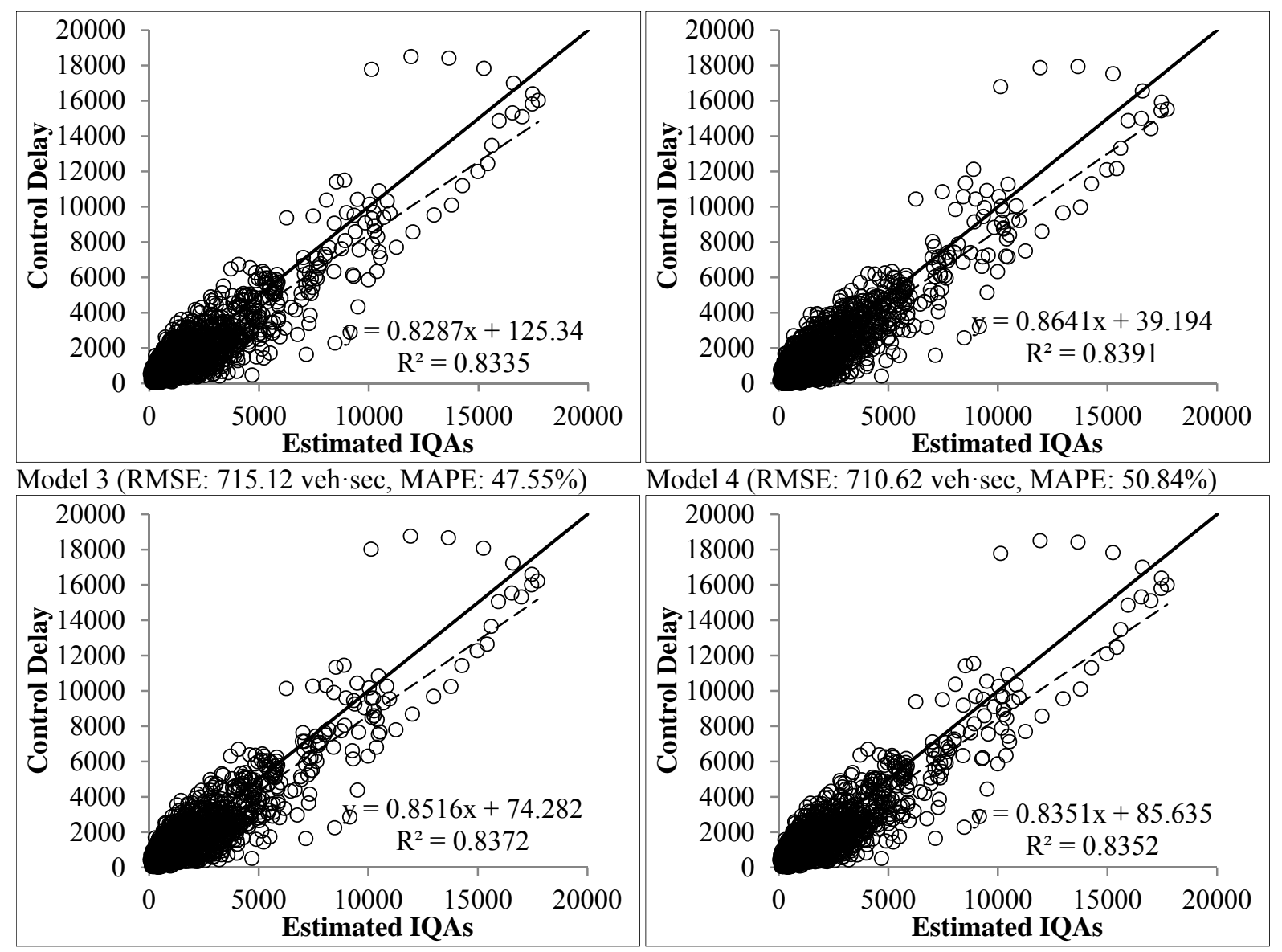

Model 5 (RMSE: $710.59 \mathrm{veh} \cdot \mathrm{sec}$, MAPE: 46.23\%) Model 6 (RMSE: $715.40 \mathrm{veh} \cdot \mathrm{sec}$, MAPE: 46.05\%)

Figure 10. Scatter plots of the six proposed models.

The estimates of all of the proposed methods are densely distributed on the periphery of their linear lines, and their $\mathrm{R}^{2}$ values are also very similar (within \pm 0.005 ). Only a slight difference in outliers can be seen between the proposed models, and the RMSE values are scattered from 704 to $715 \mathrm{veh} \cdot \mathrm{s}$ among the models. The MAPE values are also distributed from $46 \%$ to $51 \%$. The proposed model 6 shows the lowest MAPE, which means that the control delay is estimated well by model 6 under low-traffic flow conditions. We made the difficult decision that models 5 and 6 were the appropriate models for the development of group-based ATCS because they have the lowest MAPE values. Because the value of MAPE is a comparatively reliable and general standard with which to evaluate the performance of forecasting models considering the size of observed values. The results of the developed models 5 and 6 according to the number of the future time windows are described in Table 4.

Table 4 . Values of statistics of models 5 and 6 according to time windows.

\begin{tabular}{|c|c|c|c|c|c|c|c|c|c|c|}
\hline & \multicolumn{4}{|c|}{ Model 5 } & \multicolumn{5}{c|}{ Model 6 } \\
\cline { 2 - 12 } & $x=1$ & $x=2$ & $x=3$ & $x=4$ & $x=5$ & $x=1$ & $x=2$ & $x=3$ & $x=4$ & $x=5$ \\
\hline $\mathrm{R}^{2}$ & 0.88 & 0.871 & 0.861 & 0.85 & 0.837 & 0.88 & 0.871 & 0.86 & 0.849 & 0.835 \\
\hline Coefficient & 0.875 & 0.87 & 0.864 & 0.858 & 0.852 & 0.875 & 0.866 & 0.856 & 0.846 & 0.835 \\
\hline Constant & 15.52 & 29.95 & 44.2 & 58.77 & 74.28 & 15.52 & 30.48 & 46.25 & 64.03 & 85.64 \\
\hline RMSE (veh $\cdot \mathrm{s})$ & 121.3 & 251.7 & 392.4 & 544.8 & 710.6 & 121.3 & 252.3 & 394.3 & 548.2 & 715.4 \\
\hline MAPE (\%) & 83.2 & 60.69 & 51.84 & 48.01 & 46.23 & 83.2 & 60.61 & 51.84 & 47.85 & 46.05 \\
\hline
\end{tabular}

Table 4 shows that the accuracy of model 6 , with 5 cycles as the future time windows, was the highest among models, according to the MAPE values $(46.05 \%$ and $46.23 \%$, respectively), whereas the values of RMSE of both models with 1 time window cycle are the highest among the models (121.3 veh $\cdot \mathrm{sec}$ both). Because the size of the observed control delay presents a great contrast between 
the sets of different time windows - up to five times - the RMSE and MAPE values can differ greatly. The $\mathrm{R}^{2}$, coefficient, and MAPE values tend to decrease with an increase in the number of future time windows, whereas the constant and RMSE values tend to increase with an increase in the number of future time windows.

\section{CASE STUDY}

In the case study, the developed estimation approaches were applied to a real junction, which involved a sample dataset for Lankershim Boulevard in Los Angeles, USA, as provided by the Federal Highway Administration's Next Generation Simulation (NGSIM) project. This project is explained in further detail on the NGSIM website. The dataset was collected on June 16, 2005 from 8:28 a.m. to 9:00 a.m. using five video cameras. The target junction was the No. 2 intersection, where Lankershim Boulevard meets Campo De Cahuenga Way and Universal Hollywood Drive. An aerial photograph and a layout configuration of the target junction are shown in Figures 11(a) and 11(b), respectively. The recorded period had a total of 20 cycles in 6 lanes, with a cycle length of 100 seconds. The first and the last cycle were used as the warming-up and warming-down period, respectively. The lane-based observed queue lengths were directly extracted from the provided vehicle trajectory data (i.e., Vehicle ID, Frame ID, Local X and Y, Vehicle length, Velocity, Acceleration, Lane and Direction) to validate the effectiveness of the proposed IQA method and the rolling-horizon procedure.

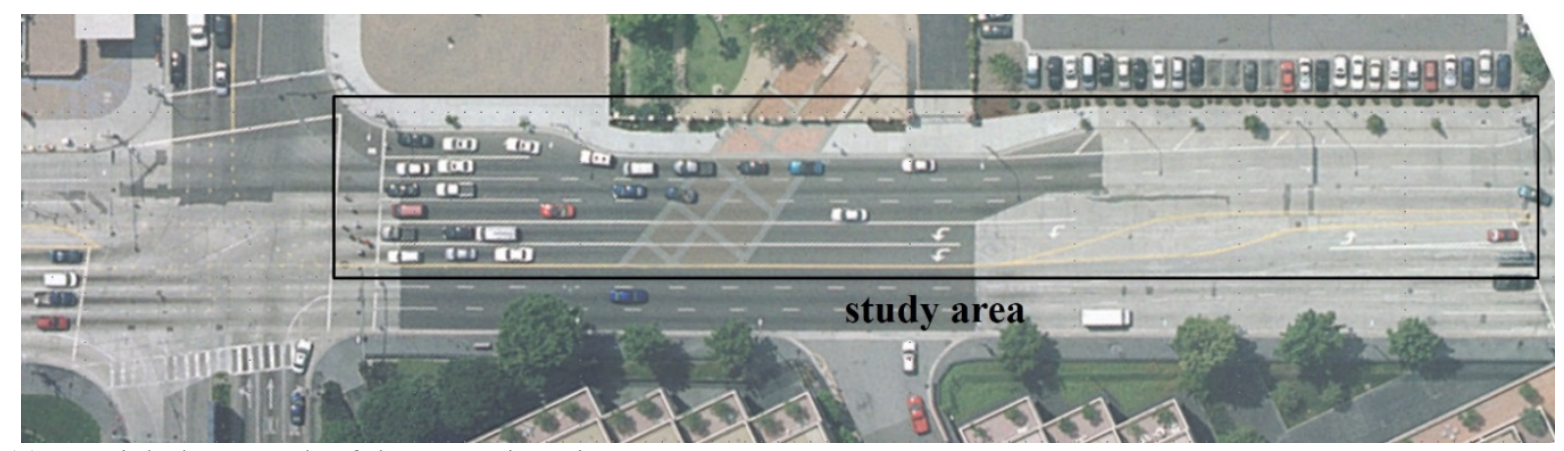

(a) Aerial photograph of the target junction

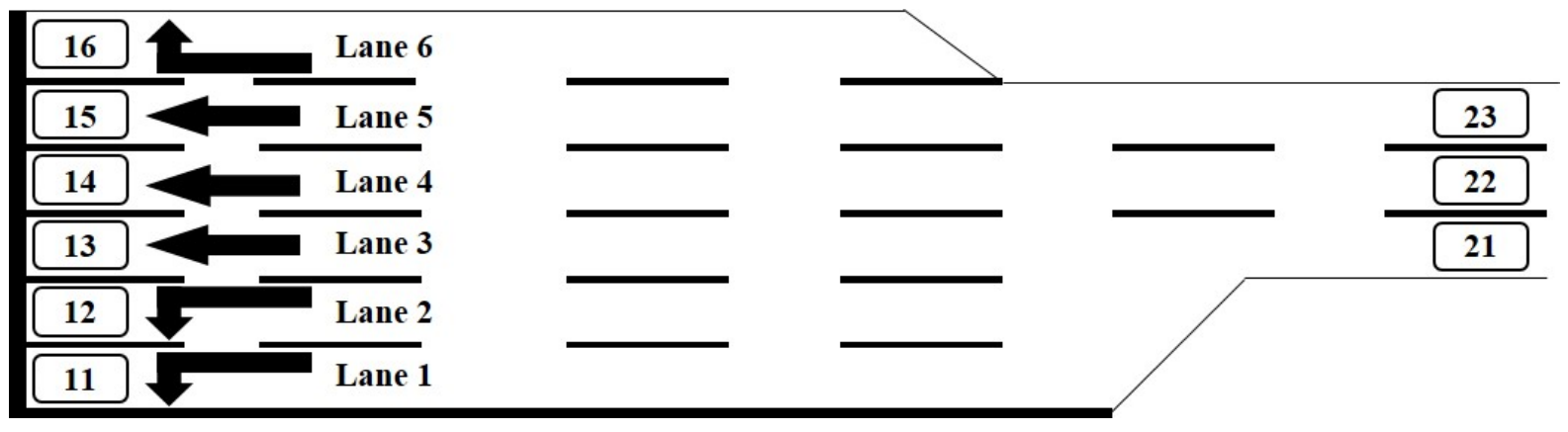

(b) Configuration of the target junction

Figure 11. Geometric conditions of the target intersection.

Scatter plots of the IQAs with adjustment factors from the proposed estimation method and from the rolling-horizon procedure for predicting lane-based IQAs are illustrated in Figure 12(a) and 12(b), respectively. 


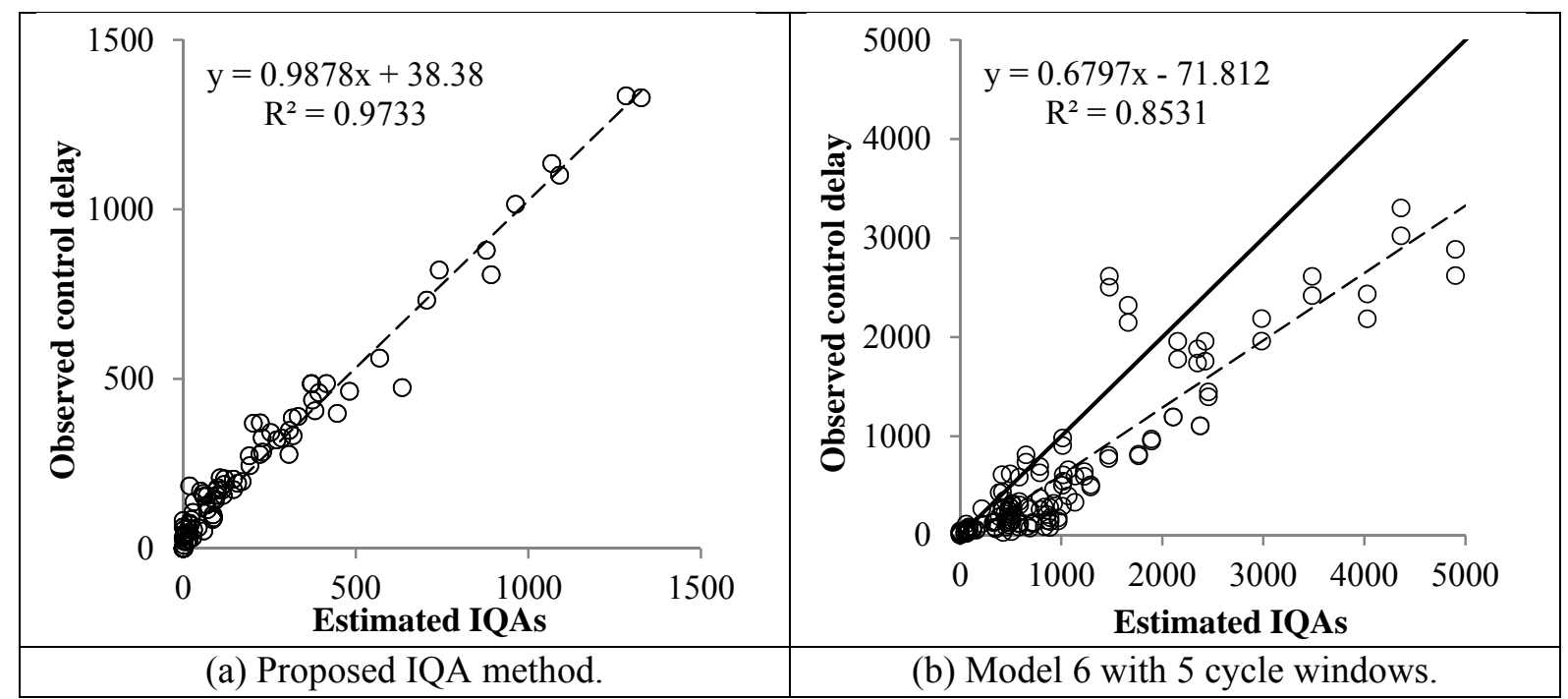

Figure 12. Scatter plot of estimates and predictions of IQA.

In Figure 12(a), the estimates from the developed models tend to almost exactly agree with the cycle measurements. Although the IQA estimates are distributed around the diagonal line with a high $\mathrm{R}^{2}$ value, 0.9733 , the scattering of estimates around the line is not narrow, with a relatively high constant, $38.38 \mathrm{veh} \cdot \mathrm{s}$, especially in low-traffic-volume conditions. The root mean square error (RMSE) and mean absolute percent error (MAPE) are $54.00 \mathrm{veh} \cdot \mathrm{s}$ and $25.78 \%$, respectively. For the rolling-horizon procedure, we used Model 6 with 5 cycles as time windows to predict the future control delay, as illustrated in Figure 12(b). The predicted values of the control delay in individual lanes are densely distributed on the periphery of the line. Only a slight difference in outliers can be seen between the proposed models, with a high $\mathrm{R}^{2}$ value, 0.8531 . The model's RMSE and MAPE values are $610.91 \mathrm{veh} \cdot \mathrm{sec}$ and $51.14 \%$, respectively. According to these statistics, the proposed methods performed well in the real situations, with a similar level of accuracy as in the simulation sets.

\section{CONCLUSIONS}

In this study, a group-based approach for the estimation of lane-based IQAs and a lane-based predictive model of control delay are proposed. The key challenges were to identify the most appropriate IQAs on the basis of group-based variables for individual lanes to the queueing formation patterns and to establish the rolling horizon procedure to predict the future components of lane-based IQAs in the time windows. The temporal and spatial information was collected on the basis of the estimated lane-based queue lengths from our previous research to estimate lane-based IQAs. In addition, we interpreted the given signal plan as group-based variables, including the start and duration of the effective green time and the cycle time. These collected and interpreted data were used to develop the mathematical structure of the control delay. Adjustment factors were developed to identify the characteristics of the control delay in a specific cycle and were applied to the mathematical structure. The variables and parameters in the developed estimation method for control delay were used in the predictive step for future delay. We constructed the rolling horizon procedure on the basis of Kalman filters with the appropriate time windows. Lane-based queue lengths at an inflection point and adjustment factors in the previous cycle were used to estimate the adjustment factors, arrival rates, and discharge rates in the next cycle, and we assumed that the current cycle was used as the calculation time for the proposed procedures.

In the simulations, we examined the performance of the proposed IQA methods and calibrated the Kalman system parameters on the basis of the estimated lane-based queue lengths. In addition, we examined six models, including the basic form and the most complicated form of the proposed method, to validate the robustness and accuracy of the proposed method. Even if the use of adjustment factors in the estimation method of control delay did not wield great influence upon the 
accuracy of estimates in all traffic flow conditions, they must be considered to identify the characteristic of control delay for the robustness of the proposed model, and they have a great effect on the increase in the accuracy of the proposed model in undersaturated traffic conditions. In the predictive processing, we considered three kinds of recursive procedure; with the direct use of past data, application of the proposed Kalman filter in the nearest future cycle only, and application of the proposed model in all future cycles. The accuracy of the proposed model 6 , including adjustment factors and all Kalman filters, was slightly better than the other proposed model according to the MAPE value. We examined the most appropriate time windows for the rolling horizon process with the two selected models, models 5 and 6, among the six proposed models. Even if the RMSE values increased according to the increase in the number of time windows, the MAPE values decreased according to the increase in the number of time windows. These two evaluation factors depend on the size of the estimates, and it was difficult to decide how many cycles should be considered in the future time windows. According to the MAPE value, which is a comparatively reliable standard for forecasting procedures, five cycles need to be considered as the most appropriate time windows for the proposed methods. However, the most appropriate range of time windows for predictive procedures depends on the characteristics of the recent fluctuations in traffic conditions.

The results of the simulations and the case study demonstrate that the proposed method is robust and accurate for the estimation of lane-based IQAs and the predictive modeling of the control delay in each cycle, which is a relatively short analysis period, based on group-based variables under a wide range of traffic conditions. The method of estimating the control delay with adjustment factors plays a significant role in increasing the accuracy of the proposed model and in classifying the queueing patterns in a specific cycle. Moreover, the calibrated Kalman models enhance the accuracy of the predictions by minimizing the error terms caused by the fluctuation in traffic flow. These proposed models will be available for use in future studies as performance indexes and for the rollinghorizon process in each cycle in optimization procedures of group-based ATCs for both isolated signalized junctions and area traffic control.

\section{Acknowledgements}

The work described in this paper was jointly supported by a Research Postgraduate Studentship and grants from the University Research Committee of the University of Hong Kong (201411159005).

\section{References}

Aboudolas, K., Papageorgiou, M., Kosmatopoulos, E., 2009. Store-and-forward based methods for the signal control problem in large-scale congested urban road networks. Transportation Research Part C: Emerging Technologies 17, 163-174.

Akçelik, R., 1980. Time-dependent expressions for delay, stop rate and queue length at traffic signals. Australian Road Research Board Melbourne, Australia.

Allsop, R. E., 1971. Delay-minimizing settings for fixed-time traffic signals at a single road junction. IMA Journal of Applied Mathematics 8, 164-185.

Allsop, R. E., 1972. Estimating the traffic capacity of a signalized road junction. Transportation Research 6, 245-255.

Bang, K. L., Nilsson, L. E., 1976. Optimal control of isolated traffic signals, Proceedings of the International Federation of Automatic Control/International Foundation for Information Process/International Federation of Oper Research Society International Symposium: Control in Transportation Systems, Columbus, Ohio.

Boillot, F., Midenet, S., Pierrelée, J. C., 2006. The real-time urban traffic control system CRONOS: Algorithm and experiments. Transportation Research Part C: Emerging Technologies 14, 1838.

D'ans, G. C., Gazis, D. C., 1976. Optimal control of oversaturated store-and-forward transportation networks. Transportation Science 10, 1-19.

Dunne, M.C., Potts, R.B., 1964. Algorithm for traffic control. Operations Research 12, 870-881. 
Farges, J. L., Henry, J. J., Tuffal, J., 1983. The PRODYN real-time traffic algorithm. In 4th IFAC Symposium on Control of Transportation Systems, Baden Baden, Germany.

Gartner, N. H., 1983. OPAC: A demand-responsive strategy for traffic signal control. Transportation Research Record: Journal of the Transportation Research Board 906, 65-72.

Gazis, D. C., 1964. Optimum control of a system of oversaturated intersections. Operations Research $12,815-831$.

Gazis D. C., Potts R. B., 1965. The oversaturated intersection. Proceedings of the Second International Symposium on the Theory of Traffic Flow, OECD, Paris, 221-237.

Ge, Y. E, Zhou, X., 2012. An alternative definition of dynamic user optimum on signalised road networks. Journal of Advanced Transportation 46, 236-253.

Gordon, R. L., Tighe, W., Siemens ITS, 2005. Traffic control systems handbook. U.S. Department of Transportation, Federal Highway Administration, Office of Operations.

Grafton, R. B., Newell, G. F., 1967. Optimal policies for the control of an undersaturated intersection. In Proceedings of the Third International Symposium on the Theory of Traffic Flow. New York.

Green, D. H., 1967. Control of oversaturated intersections. Operational Research Quarterly 18, 161173.

Han, K., Gayah, V. V., Piccoli, B., Friesz, T. L., Yao, T., 2014. On the continuum approximation of the on-and-off signal control on dynamic traffic networks. Transportation Research Part B: Methodological 61, 73-97.

Heydecker, B. G., Dudgeon, I. W., 1987. Calculation of signal settings to minimise delay at a junction. In Proceedings of the 10th International Symposium on Transportation and Traffic Theory, MIT, July, Elsevier, New York, 159-178.

Heydecker, B. G., 1992. Sequencing of traffic signals. In Griffiths, J. D. (Ed.), Mathematics in Transport and Planning and Control. Clarendon Press, Oxford, U.K., 57-67.

Highway Capacity Manual, 2010. TRB, National Research Council, Washington D.C.

Hunt, P. B., Robertson, D. I., Bretherton, R. D., Winton, R. I., 1981. SCOOT - A traffic responsive method of coordinating signals. LR1014, Transport and Road Research Laboratory, Crowthorne, U.K.

Lee, S., Wong, S. C., Pang, C. C. C., Choi, K., 2015a. Real-time estimation of lane-to-lane turning flows at isolated signalized junctions. Intelligent Transportation Systems, IEEE Transactions on $16,1549-1558$.

Lee, S., Wong, S. C., Li, Y. C., 2015b. Real-time estimation of lane-based queue lengths at isolated signalized junctions. Transportation Research Part C: Emerging Technologies 56, 1-17.

Lighthill M. J., Whitham G. B., 1955. On kinematic waves. I: Flood movement in long rivers. II: A theory of traffic flow on long crowded roads. In Proceedings of the Royal Society of London A: Mathematical, Physical and Engineering Sciences, London, A229, 281-345.

Lindley, D.V., 1952. The theory of queues with a single server, Mathematical Proceedings of the Cambridge Philosophical Society. Cambridge University Press, 277-289.

Luyanda, F., Gettman, D., Head, L., Shelby, S., Bullock, D., Mirchandani, P., 2003. ACS-Lite algorithmic architecture: applying adaptive control system technology to closed-loop traffic signal control systems. Transportation Research Record: Journal of the Transportation Research Board 1856, 175-184.

Morris, R. W., Pak-Poy, P. G., 1967. Intersection control by vehicle actuated signals. Traffic Engineering \& Control 9, 288-293

Miller, A. J., 1963. A computer control system for traffic networks. In Proceedings of the Second International Symposium on the Theory of Traffic Flow, London, 200-220.

Michalopoulos, P. G., Stephanopoulos, G., 1977a. Oversaturated signal systems with queue length constraints. I: Single intersection. Transportation Research 11, 413-421.

Michalopoulos, P. G., Stephanopoulos, G., 1977b. Oversaturated signal systems with queue length constraints. II: Systems of intersections. Transportation Research 11, 423-428.

Mirchandani, P. B., Head, L., 2001. A real-time traffic signal control system: architecture, algorithms, and analysis. Transportation Research Part C: Emerging Technologies 9, 415-432.

Richards, P. I., 1956. Shock waves on the highway. Operations Research 4, 42-51.

Rosdolsky, H. G., 1973. A method for adaptive traffic control. Transportation Research 7, 1-16. 
Ross, D. W., Sandys, R. C., Schlaefli, J. L., 1971. A computer control scheme for critical-intersection control in an urban network. Transportation Science 5, 141-160.

Saaty, T. L., 1961. Elements of queueing theory: With applications. McGraw-Hill, New York.

Silcock, J. P., 1997. Designing signal-controlled junctions for group-based operation. Transportation Research Part A: Policy and Practice 31, 157-173.

Smith, M. J., 1979a. The existence, uniqueness and stability of traffic equilibria. Transportation Research Part B: Methodological 13, 295-304.

Smith, M. J., 1979b. Traffic control and route-choice; a simple example. Transportation Research Part B: Methodological 13, 289-294.

Smith, M. J., 1981. Properties of a traffic control policy which ensure the existence of a traffic equilibrium consistent with the policy. Transportation Research Part B: Methodological 15, 453-462.

Smith, M. J., 1983. The existence and calculation of traffic equilibria. Transportation Research Part B: Methodological 17, 291-303.

Smith, M. J., 2011. Dynamics of route choice and signal control in capacitated networks. Journal of Choice Modelling 4, 30-51.

Smith, M. J., Ghali, M., 1990. The dynamics of traffic assignment and traffic control: A theoretical study. Transportation Research Part B: Methodological 24, 409-422.

Smith, M. J., Mounce, R., 2015. Traffic control and route choice; capacity maximization and stability. In 21 st International Symposium on Transportation and Traffic Theory, Kobe, Japan.

Smith, M. J., Van Vuren, T., 1993. Traffic equilibrium with responsive traffic control. Transportation Science 27, 118-132.

Varaiya, P., 2013. Max pressure control of a network of signalized intersections. Transportation Research Part C: Emerging Technologies 36, 177-195.

Webster, F. V., 1958. Traffic signal settings, road research technical paper no. 39. Road Research Laboratory.

Wong, C. K., Wong, S. C., 2003a. A lane-based optimization method for minimizing delay at isolated signal-controlled junctions. Journal of Mathematical Modelling and Algorithms 2, 379-406.

Wong, C. K., Wong, S. C., 2003b. Lane-based optimization of signal timings for isolated junctions. Transportation Research Part B: Methodological 37, 63-84.

Wong, C. K., Wong, S. C., Tong, C. O., 2006. A lane-based optimization method for the multi-period analysis of isolated signal-controlled junctions. Transportmetrica 2, 53-85.

Wong, S. C., 1995. Derivatives of the performance index for the traffic model from TRANSYT. Transportation Research Part B: Methodological 29, 303-327.

Wong, S. C., 1996. Group-based optimisation of signal timings using the TRANSYT traffic model. Transportation Research Part B: Methodological 30, 217-244.

Wong, S. C., Yang, C., 1999. An iterative group-based signal optimization scheme for traffic equilibrium networks. Journal of Advanced Transportation 33, 201-217.

Wong, S. C., Yang, H., 1997. Reserve capacity of a signal-controlled road network. Transportation Research Part B: Methodological 31, 397-402. 


\section{Appendix I}

\begin{tabular}{|c|c|c|c|c|}
\hline$\hat{q}_{k}^{n}\left(\alpha_{k}^{n}\right)$ & $\hat{q}_{k}^{n}\left(\frac{\theta_{k}^{n}}{\zeta^{n}}+\beta_{k}^{n}\right)$ & $\hat{q}_{k}^{n}\left(\frac{\theta_{k}^{n}+\phi_{k}^{n}}{\zeta^{n}}+\gamma_{k}^{n}\right)$ & $\hat{q}_{k}^{n}\left(\frac{\theta_{k}^{n}+\phi_{k}^{n}}{\zeta^{n}}+\gamma_{k}^{n}+\delta_{k}^{n}\right)$ & $\hat{q}_{k}^{n}\left(\frac{1}{\zeta^{n}}\right)$ \\
\hline $\mathrm{q} 1$ & $\mathrm{q} 2$ & $\mathrm{q} 3$ & $\mathrm{q} 4$ & $\mathrm{q} 5$ \\
\hline
\end{tabular}

\begin{tabular}{|c|c|c|c|c|}
\hline \multicolumn{5}{|c|}{$\Omega_{k}^{n}$} \\
\hline$\alpha_{k}^{n}$ & $\beta_{k}^{n}$ & $\gamma_{k}^{n}$ & $\gamma_{k}^{n}$ & $\delta_{k}^{n}$ \\
\hline $\mathrm{f} 1$ & $\mathrm{f} 2$ & $\mathrm{f3}$ & $\mathrm{f} 4$ & $\mathrm{f5}$ \\
\hline
\end{tabular}

\begin{tabular}{|c|c|c|c|c|c|c|c|c|c|c|c|c|c|c|}
\hline \multirow{2}{*}{$V$} & \multirow{2}{*}{$x$} & & \multicolumn{3}{|c|}{ Approach 1} & \multicolumn{3}{|c|}{ Approach 2} & \multicolumn{3}{|c|}{ Approach 3} & \multicolumn{3}{|c|}{ Approach 4} \\
\hline & & & lane 1 & lane 2 & lane 3 & lane 1 & lane 2 & lane 3 & lane 1 & lane 2 & lane 3 & lane 1 & lane 2 & lane 3 \\
\hline \multirow{20}{*}{ q1 } & \multirow{4}{*}{1} & A & 0.82 & 0.80 & 0.94 & 0.76 & 0.82 & 0.86 & 0.77 & 0.67 & 0.70 & 0.69 & 0.68 & 0.76 \\
\hline & & $\mathbf{Q}$ & 4.31 & 3.71 & 7.24 & 3.25 & 2.38 & 4.44 & 1.37 & 0.86 & 2.31 & 0.50 & 0.73 & 1.22 \\
\hline & & $\mathbf{H}$ & 0.82 & 0.81 & 0.94 & 0.76 & 0.82 & 0.85 & 0.77 & 0.67 & 0.67 & 0.69 & 0.68 & 0.75 \\
\hline & & $\mathbf{R}$ & 4.28 & 3.72 & 7.23 & 3.24 & 2.37 & 4.40 & 1.37 & 0.86 & 2.20 & 0.50 & 0.73 & 1.21 \\
\hline & \multirow{4}{*}{2} & A & 0.80 & 0.78 & 0.92 & 0.72 & 0.78 & 0.80 & 0.68 & 0.65 & 0.66 & 0.60 & 0.53 & 0.69 \\
\hline & & $\mathbf{Q}$ & 4.97 & 4.14 & 10.11 & 3.82 & 3.01 & 6.08 & 1.86 & 0.91 & 2.76 & 0.61 & 0.98 & 1.54 \\
\hline & & $\mathbf{H}$ & 0.79 & 0.78 & 0.92 & 0.72 & 0.77 & 0.80 & 0.68 & 0.65 & 0.64 & 0.60 & 0.53 & 0.69 \\
\hline & & $\mathbf{R}$ & 4.91 & 4.17 & 10.11 & 3.80 & 2.97 & 6.06 & 1.85 & 0.90 & 2.70 & 0.60 & 0.97 & 1.53 \\
\hline & \multirow{4}{*}{3} & A & 0.80 & 0.76 & 0.89 & 0.69 & 0.75 & 0.78 & 0.66 & 0.63 & 0.63 & 0.59 & 0.52 & 0.69 \\
\hline & & $\mathbf{Q}$ & 4.98 & 4.39 & 14.03 & 4.25 & 3.33 & 6.87 & 1.94 & 0.94 & 3.07 & 0.62 & 0.98 & 1.54 \\
\hline & & $\mathbf{H}$ & 0.79 & 0.76 & 0.89 & 0.68 & 0.74 & 0.77 & 0.66 & 0.63 & 0.60 & 0.59 & 0.52 & 0.69 \\
\hline & & $\mathbf{R}$ & 4.92 & 4.39 & 13.98 & 4.20 & 3.28 & 6.82 & 1.93 & 0.93 & 2.92 & 0.62 & 0.98 & 1.54 \\
\hline & \multirow{4}{*}{4} & $\mathbf{A}$ & 0.81 & 0.77 & 0.87 & 0.72 & 0.73 & 0.78 & 0.66 & 0.63 & 0.62 & 0.61 & 0.44 & 0.65 \\
\hline & & $\mathbf{Q}$ & 4.71 & 4.25 & 16.45 & 3.98 & 3.58 & 6.96 & 2.00 & 0.95 & 3.15 & 0.60 & 1.10 & 1.74 \\
\hline & & $\mathbf{H}$ & 0.80 & 0.77 & 0.87 & 0.70 & 0.72 & 0.76 & 0.65 & 0.62 & 0.56 & 0.61 & 0.44 & 0.63 \\
\hline & & $\mathbf{R}$ & 4.60 & 4.28 & 16.44 & 3.87 & 3.51 & 6.77 & 1.97 & 0.95 & 2.84 & 0.60 & 1.10 & 1.67 \\
\hline & \multirow{4}{*}{5} & A & 0.80 & 0.77 & 0.83 & 0.76 & 0.73 & 0.78 & 0.61 & 0.61 & 0.62 & 0.57 & 0.39 & 0.63 \\
\hline & & $\mathbf{Q}$ & 5.03 & 4.25 & 20.31 & 3.52 & 3.60 & 7.13 & 2.21 & 1.00 & 3.23 & 0.65 & 1.16 & 1.84 \\
\hline & & $\mathbf{H}$ & 0.78 & 0.78 & 0.83 & 0.74 & 0.71 & 0.75 & 0.60 & 0.60 & 0.53 & 0.56 & 0.39 & 0.60 \\
\hline & & $\mathbf{R}$ & 4.89 & 4.28 & 20.28 & 3.41 & 3.52 & 6.85 & 2.16 & 0.99 & 2.78 & 0.64 & 1.16 & 1.74 \\
\hline
\end{tabular}

\begin{tabular}{|c|c|c|c|c|c|c|c|c|c|c|c|c|c|c|}
\hline \multirow[b]{2}{*}{ ( } & \multirow{2}{*}{$x$} & & \multicolumn{3}{|c|}{ Approach 1} & \multicolumn{3}{|c|}{ Approach 2} & \multicolumn{3}{|c|}{ Approach 3} & \multicolumn{3}{|c|}{ Approach 4} \\
\hline & & & lane 1 & lane 2 & lane 3 & lane 1 & lane 2 & lane 3 & lane 1 & lane 2 & lane 3 & lane 1 & lane 2 & lane 3 \\
\hline \multirow{4}{*}{\multicolumn{2}{|c|}{1}} & A & 0.81 & 0.79 & 0.94 & 0.80 & 0.85 & 0.87 & 0.81 & 0.76 & 0.79 & 0.83 & 0.85 & 0.87 \\
\hline & & $\mathbf{Q}$ & 4.56 & 3.92 & 7.31 & 5.05 & 3.44 & 6.54 & 5.05 & 3.35 & 7.64 & 4.35 & 3.07 & 6.19 \\
\hline & & $\mathbf{H}$ & 0.80 & 0.79 & 0.94 & 0.79 & 0.85 & 0.87 & 0.81 & 0.76 & 0.77 & 0.83 & 0.85 & 0.87 \\
\hline & & $\mathbf{R}$ & 4.53 & 3.93 & 7.31 & 5.04 & 3.44 & 6.50 & 5.04 & 3.35 & 7.45 & 4.35 & 3.06 & 6.14 \\
\hline \multirow{7}{*}{ q2 } & & A & 0.79 & 0.76 & 0.92 & 0.77 & 0.81 & 0.83 & 0.73 & 0.72 & 0.78 & 0.78 & 0.82 & 0.86 \\
\hline & , & $\mathbf{Q}$ & 5.14 & 4.28 & 10.11 & 5.70 & 4.38 & 8.54 & 6.86 & 3.83 & 8.22 & 5.60 & 3.76 & 6.62 \\
\hline & & $\mathbf{H}$ & 0.78 & 0.77 & 0.92 & 0.77 & 0.80 & 0.83 & 0.73 & 0.72 & 0.77 & 0.77 & 0.81 & 0.86 \\
\hline & & $\mathbf{R}$ & 5.09 & 4.31 & 10.12 & 5.65 & 4.34 & 8.54 & 6.88 & 3.82 & 8.11 & 5.58 & 3.75 & 6.61 \\
\hline & & A & 0.78 & 0.76 & 0.89 & 0.75 & 0.79 & 0.81 & 0.73 & 0.76 & 0.78 & 0.78 & 0.81 & 0.87 \\
\hline & 3 & $\mathbf{Q}$ & 5.24 & 4.43 & 14.09 & 6.38 & 4.72 & 9.62 & 6.97 & 3.45 & 8.45 & 5.64 & 3.84 & 6.25 \\
\hline & & $\mathbf{H}$ & 0.77 & 0.76 & 0.88 & 0.73 & 0.78 & 0.80 & 0.73 & 0.75 & 0.76 & 0.77 & 0.81 & 0.87 \\
\hline
\end{tabular}




\begin{tabular}{|c|c|c|c|c|c|c|c|c|c|c|c|c|c|}
\hline & $\mathbf{R}$ & 5.19 & 4.44 & 14.04 & 6.27 & 4.66 & 9.59 & 6.94 & 3.40 & 8.24 & 5.61 & 3.83 & 6.26 \\
\hline \multirow{4}{*}{4} & A & 0.80 & 0.76 & 0.86 & 0.76 & 0.78 & 0.80 & 0.73 & 0.76 & 0.79 & 0.78 & 0.78 & 0.88 \\
\hline & $\mathbf{Q}$ & 4.95 & 4.26 & 16.60 & 6.10 & 5.00 & 10.17 & 7.07 & 3.32 & 8.17 & 5.60 & 4.34 & 6.47 \\
\hline & $\mathbf{H}$ & 0.78 & 0.77 & 0.86 & 0.74 & 0.77 & 0.79 & 0.71 & 0.77 & 0.76 & 0.77 & 0.78 & 0.86 \\
\hline & $\mathbf{R}$ & 4.86 & 4.30 & 16.59 & 5.93 & 4.90 & 10.00 & 6.88 & 3.34 & 7.81 & 5.49 & 4.32 & 6.33 \\
\hline \multirow{4}{*}{5} & $\mathbf{A}$ & 0.79 & 0.77 & 0.83 & 0.79 & 0.78 & 0.81 & 0.74 & 0.75 & 0.80 & 0.79 & 0.77 & 0.88 \\
\hline & $\mathbf{Q}$ & 5.24 & 4.22 & 20.33 & 5.58 & 5.08 & 9.99 & 7.06 & 3.52 & 8.46 & 5.57 & 4.51 & 6.46 \\
\hline & $\mathbf{H}$ & 0.77 & 0.77 & 0.83 & 0.77 & 0.76 & 0.79 & 0.71 & 0.75 & 0.74 & 0.77 & 0.77 & 0.86 \\
\hline & $\mathbf{R}$ & 5.12 & 4.25 & 20.29 & 5.40 & 4.98 & 9.76 & 6.84 & 3.55 & 7.88 & 5.45 & 4.50 & 6.31 \\
\hline
\end{tabular}

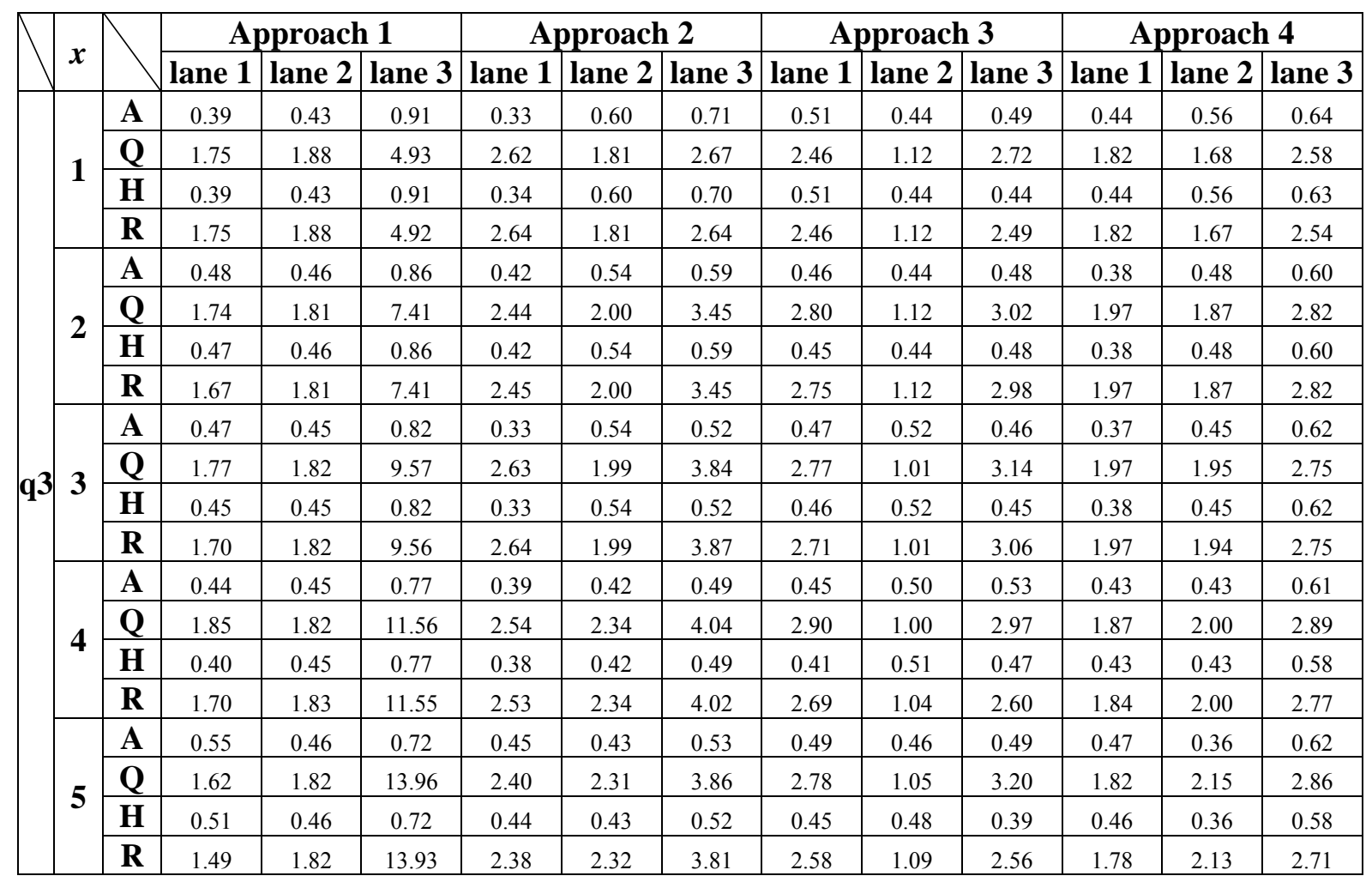

\begin{tabular}{|c|c|c|c|c|c|c|c|c|c|c|c|c|c|c|}
\hline & \multirow[b]{2}{*}{$x$} & & \multicolumn{3}{|c|}{ Approach 1} & \multicolumn{3}{|c|}{ Approach 2} & \multicolumn{3}{|c|}{ Approach 3} & \multicolumn{3}{|c|}{ Approach 4} \\
\hline & & & lane 1 & lane 2 & lane 3 & \begin{tabular}{|l|} 
lane 1 \\
\end{tabular} & lane 2 & lane 3 & lane 1 & lane 2 & lane 3 & lane 1 & lane 2 & lane 3 \\
\hline \multirow{8}{*}{\multicolumn{2}{|c|}{1}} & A & 0.11 & 0.15 & 0.92 & 0.32 & 0.60 & 0.73 & 0.60 & 0.04 & 0.56 & 0.34 & 0.40 & 0.57 \\
\hline & & $\mathbf{Q}$ & 0.15 & 0.47 & 4.12 & 0.49 & 1.05 & 1.78 & 0.51 & 0.25 & 0.94 & 1.94 & 1.92 & 2.88 \\
\hline & & $\mathbf{H}$ & 0.11 & 0.15 & 0.92 & 0.32 & 0.60 & 0.72 & 0.60 & 0.04 & 0.43 & 0.34 & 0.40 & 0.56 \\
\hline & & $\mathbf{R}$ & 0.15 & 0.47 & 4.12 & 0.49 & 1.05 & 1.76 & 0.51 & 0.25 & 0.71 & 1.94 & 1.91 & 2.84 \\
\hline & & A & 0.00 & 0.18 & 0.85 & 0.19 & 0.50 & 0.52 & 0.43 & 0.04 & 0.34 & 0.24 & 0.33 & 0.55 \\
\hline & & $\mathbf{Q}$ & 0.15 & 0.46 & 7.22 & 0.52 & 1.24 & 2.72 & 0.65 & 0.25 & 1.14 & 2.08 & 2.01 & 3.04 \\
\hline & & $\mathbf{H}$ & 0.00 & 0.18 & 0.85 & 0.19 & 0.49 & 0.52 & 0.43 & 0.04 & 0.33 & 0.24 & 0.33 & 0.56 \\
\hline & & $\mathbf{R}$ & 0.15 & 0.46 & 7.22 & 0.52 & 1.23 & 2.72 & 0.65 & 0.25 & 1.10 & 2.08 & 2.01 & 3.04 \\
\hline 44 & \multirow{4}{*}{3} & A & 0.02 & 0.41 & 0.79 & 0.03 & 0.38 & 0.40 & 0.21 & 0.13 & 0.28 & 0.29 & 0.38 & 0.54 \\
\hline & & $\mathbf{Q}$ & 0.15 & 0.40 & 9.70 & 0.54 & 1.40 & 3.13 & 0.76 & 0.24 & 1.24 & 2.02 & 1.94 & 3.08 \\
\hline & & $\mathbf{H}$ & 0.02 & 0.41 & 0.79 & 0.03 & 0.38 & 0.40 & 0.21 & 0.12 & 0.26 & 0.29 & 0.38 & 0.55 \\
\hline & & $\mathbf{R}$ & 0.15 & 0.40 & 9.68 & 0.54 & 1.39 & 3.13 & 0.76 & 0.24 & 1.15 & 2.03 & 1.93 & 3.09 \\
\hline & \multirow{4}{*}{4} & A & 0.12 & 0.08 & 0.74 & 0.05 & 0.26 & 0.41 & 0.21 & 0.05 & 0.20 & 0.32 & 0.32 & 0.55 \\
\hline & & $\mathbf{Q}$ & 0.13 & 0.48 & 11.75 & 0.54 & 1.53 & 3.15 & 0.77 & 0.25 & 1.24 & 1.99 & 2.04 & 3.11 \\
\hline & & $\mathbf{H}$ & 0.14 & 0.08 & 0.74 & 0.05 & 0.26 & 0.40 & 0.21 & 0.05 & 0.20 & 0.32 & 0.32 & 0.53 \\
\hline & & $\mathbf{R}$ & 0.15 & 0.48 & 11.75 & 0.54 & 1.52 & 3.10 & 0.77 & 0.25 & 1.18 & 1.96 & 2.04 & 2.98 \\
\hline
\end{tabular}




\begin{tabular}{|c|c|c|c|c|c|c|c|c|c|c|c|c|c|}
\hline \multirow{4}{*}{5} & $\mathbf{A}$ & 0.13 & 0.19 & 0.68 & 0.06 & 0.26 & 0.39 & 0.17 & 0.04 & 0.19 & 0.32 & 0.30 & 0.58 \\
\hline & $\mathbf{Q}$ & 0.13 & 0.47 & 13.91 & 0.54 & 1.54 & 3.22 & 0.78 & 0.25 & 1.26 & 2.01 & 2.09 & 3.01 \\
\hline & $\mathbf{H}$ & 0.15 & 0.19 & 0.68 & 0.06 & 0.26 & 0.38 & 0.17 & 0.04 & 0.13 & 0.31 & 0.29 & 0.55 \\
\hline & $\mathbf{R}$ & 0.15 & 0.47 & 13.91 & 0.54 & 1.53 & 3.12 & 0.78 & 0.25 & 0.91 & 1.97 & 2.07 & 2.86 \\
\hline
\end{tabular}

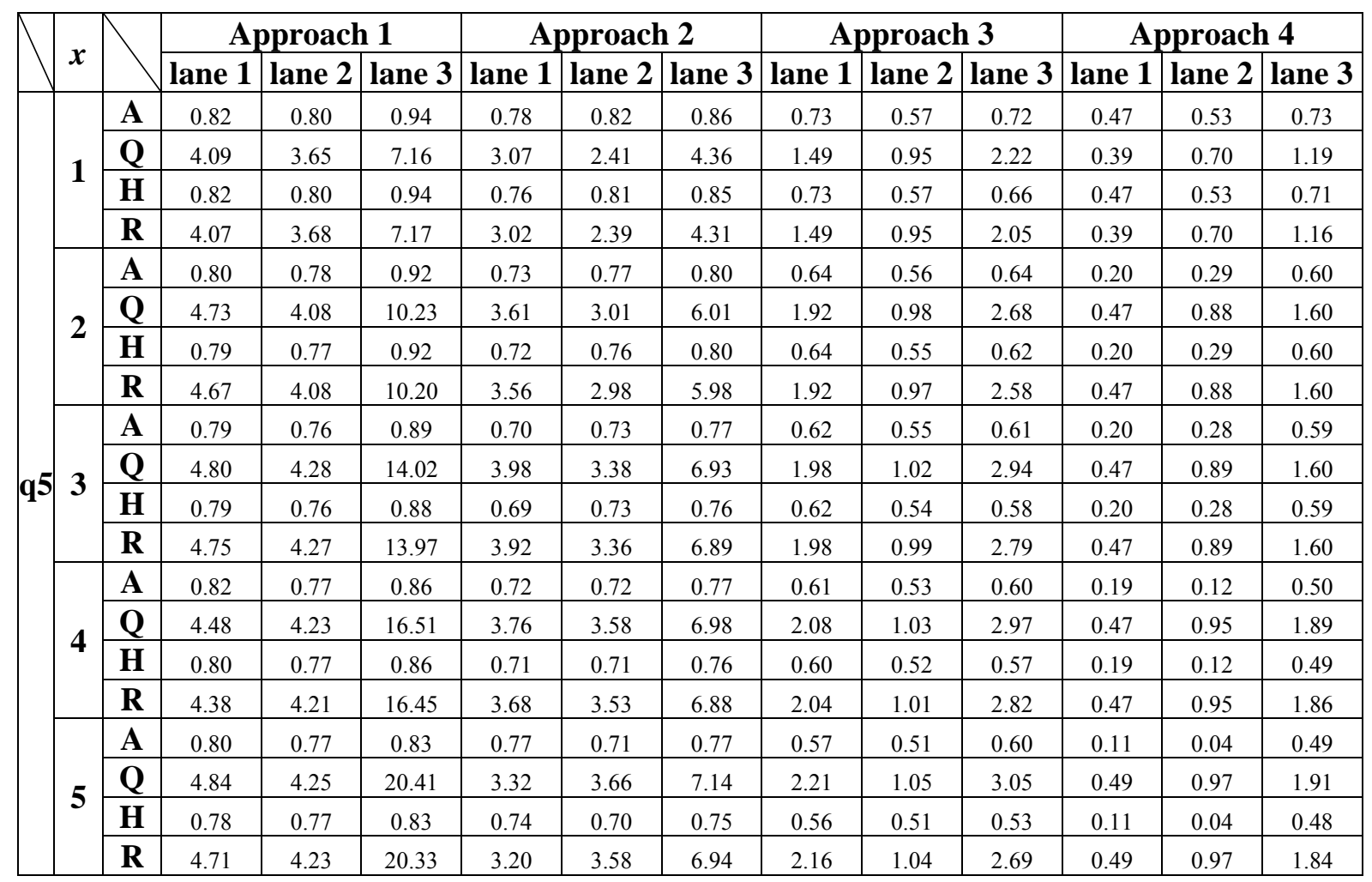

\begin{tabular}{|c|c|c|c|c|c|c|c|c|c|c|c|c|c|c|}
\hline \multirow{2}{*}{$V$} & \multirow[b]{2}{*}{$x$} & & \multicolumn{3}{|c|}{ Approach 1} & \multicolumn{3}{|c|}{ Approach 2} & \multicolumn{3}{|c|}{ Approach 3} & \multicolumn{3}{|c|}{ Approach 4} \\
\hline & & & lane 1 & lane 2 & lane 3 & lane 1 & lane 2 & lane 3 & lane 1 & lane 2 & lane 3 & lane 1 & lane 2 & lane 3 \\
\hline \multirow{20}{*}{ f1 } & \multirow{4}{*}{1} & $\mathbf{A}$ & 0.00 & 0.00 & 0.00 & 0.03 & 0.00 & 0.00 & 0.11 & 0.11 & 0.01 & 0.52 & 0.48 & 0.47 \\
\hline & & $\mathbf{Q}$ & 0.00 & 0.00 & 0.00 & 6.28 & 4.67 & 1.61 & 29.12 & 42.44 & 13.66 & 88.33 & 104.67 & 56.03 \\
\hline & & $\mathbf{H}$ & 0.00 & 0.00 & 0.00 & 0.03 & 0.00 & 0.00 & 0.11 & 0.11 & 0.01 & 0.52 & 0.48 & 0.47 \\
\hline & & $\mathbf{R}$ & 0.00 & 0.00 & 0.00 & 6.28 & 4.67 & 1.61 & 29.12 & 42.52 & 13.75 & 88.17 & 104.22 & 56.03 \\
\hline & \multirow{4}{*}{2} & $\mathbf{A}$ & 0.00 & 0.00 & 0.00 & 0.14 & 0.13 & 0.00 & 0.00 & 0.15 & 0.00 & 0.48 & 0.46 & 0.41 \\
\hline & & $\mathbf{Q}$ & 0.00 & 0.00 & 0.00 & 6.10 & 4.51 & 1.59 & 32.25 & 41.43 & 13.40 & 92.75 & 99.92 & 59.38 \\
\hline & & $\mathbf{H}$ & 0.00 & 0.00 & 0.00 & 0.14 & 0.13 & 0.00 & 0.00 & 0.15 & 0.00 & 0.48 & 0.48 & 0.41 \\
\hline & & $\mathbf{R}$ & 0.00 & 0.00 & 0.00 & 6.10 & 4.54 & 1.59 & 29.17 & 41.64 & 13.61 & 92.73 & 105.28 & 59.33 \\
\hline & \multirow{4}{*}{3} & A & 0.00 & 0.00 & 0.00 & 0.00 & 0.00 & 0.00 & 0.10 & 0.10 & 0.00 & 0.48 & 0.48 & 0.42 \\
\hline & & $\mathbf{Q}$ & 0.00 & 0.00 & 0.00 & 6.22 & 4.60 & 1.59 & 31.97 & 41.97 & 13.40 & 93.00 & 97.09 & 58.62 \\
\hline & & $\mathbf{H}$ & 0.00 & 0.00 & 0.00 & 0.00 & 0.00 & 0.00 & 0.09 & 0.10 & 0.00 & 0.48 & 0.51 & 0.42 \\
\hline & & $\mathbf{R}$ & 0.00 & 0.00 & 0.00 & 6.22 & 4.63 & 1.59 & 28.91 & 42.18 & 13.61 & 93.07 & 102.32 & 58.60 \\
\hline & \multirow{4}{*}{4} & A & 0.00 & 0.00 & 0.00 & 0.02 & 0.21 & 0.23 & 0.11 & 0.15 & 0.16 & 0.45 & 0.46 & 0.35 \\
\hline & & $\mathbf{Q}$ & 0.00 & 0.00 & 0.00 & 6.26 & 4.42 & 1.51 & 32.10 & 41.73 & 13.14 & 96.79 & 100.26 & 62.91 \\
\hline & & $\mathbf{H}$ & 0.00 & 0.00 & 0.00 & 0.02 & 0.21 & 0.23 & 0.10 & 0.15 & 0.16 & 0.45 & 0.48 & 0.35 \\
\hline & & $\mathbf{R}$ & 0.00 & 0.00 & 0.00 & 6.26 & 4.45 & 1.51 & 29.03 & 41.93 & 13.34 & 96.75 & 105.88 & 63.14 \\
\hline & \multirow{4}{*}{5} & A & 0.00 & 0.00 & 0.00 & 0.00 & 0.11 & 0.00 & 0.03 & 0.06 & 0.02 & 0.50 & 0.50 & 0.38 \\
\hline & & $\mathbf{Q}$ & 0.00 & 0.00 & 0.00 & 6.29 & 4.59 & 1.61 & 32.39 & 42.72 & 13.53 & 91.04 & 94.78 & 61.41 \\
\hline & & $\mathbf{H}$ & 0.00 & 0.00 & 0.00 & 0.00 & 0.11 & 0.00 & 0.03 & 0.06 & 0.02 & 0.50 & 0.53 & 0.38 \\
\hline & & $\mathbf{R}$ & 0.00 & 0.00 & 0.00 & 6.29 & 4.62 & 1.61 & 29.45 & 42.93 & 13.74 & 91.15 & 99.68 & 61.65 \\
\hline
\end{tabular}




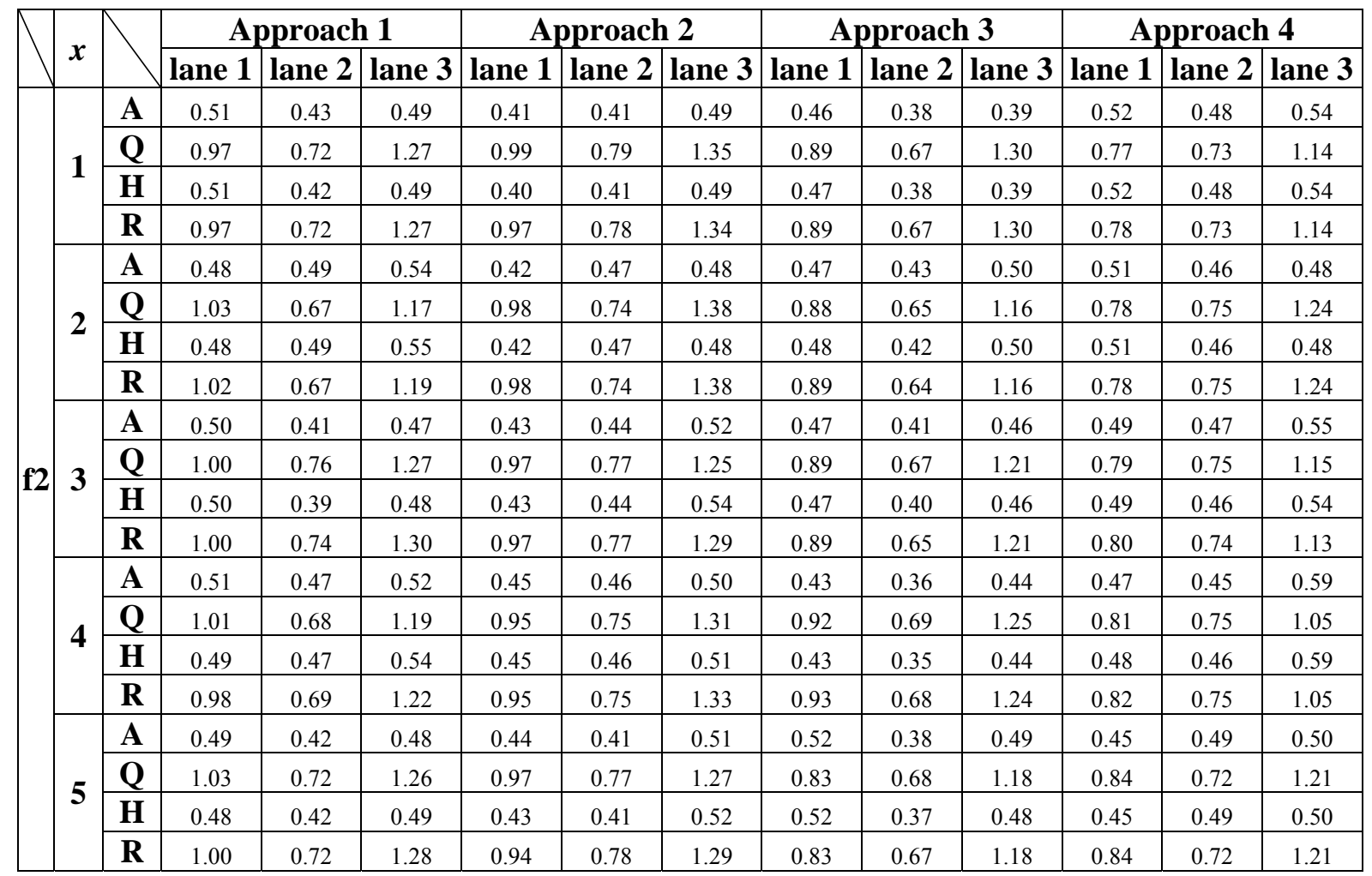

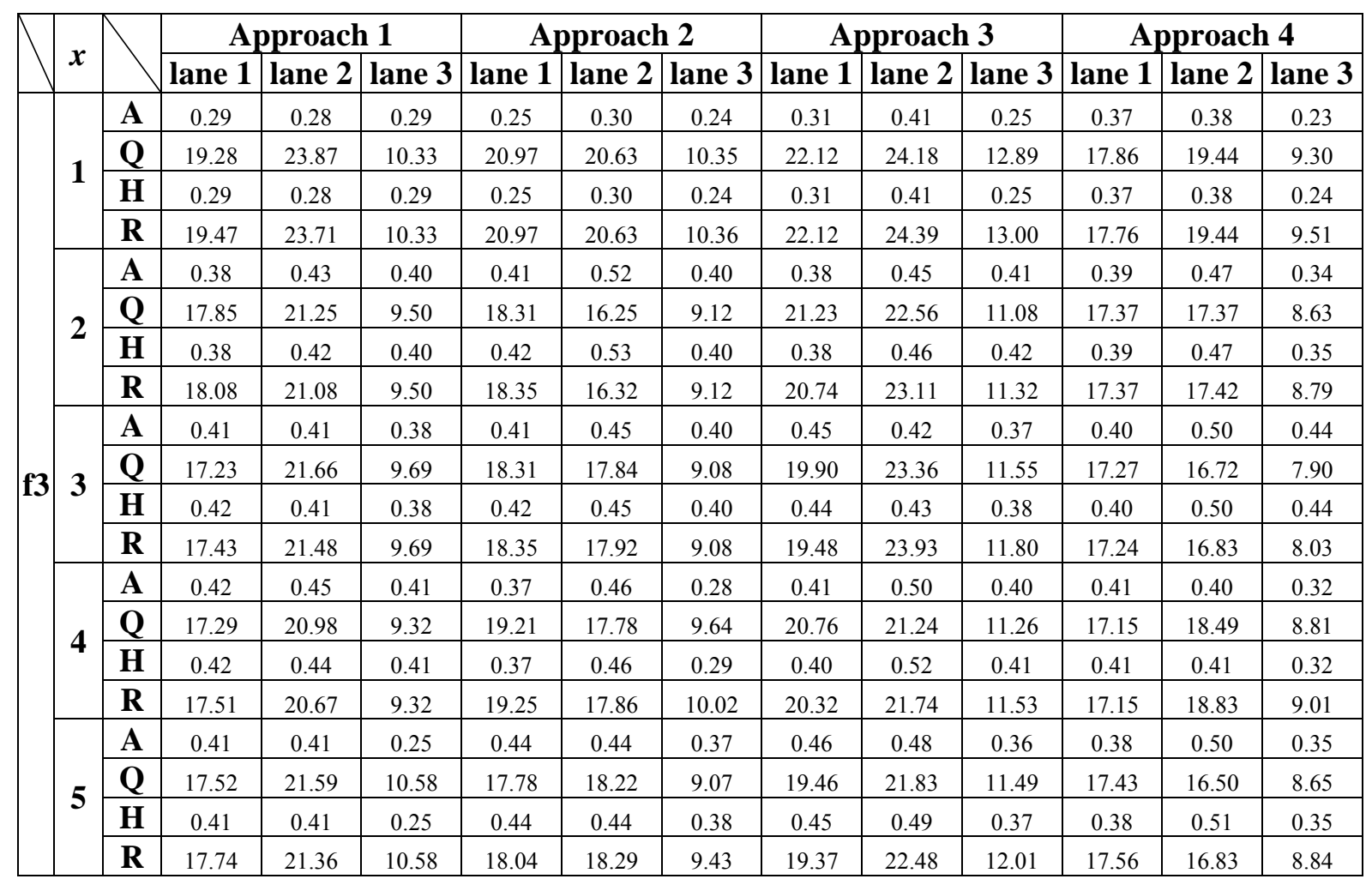

\begin{tabular}{|c|c|c|c|c|c|c|c|c|c|c|c|c|c|c|}
\hline \multirow[b]{2}{*}{ 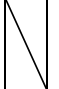 } & \multirow[b]{2}{*}{$x$} & & \multicolumn{3}{|c|}{ Approach 1} & \multicolumn{3}{|c|}{ Approach 2} & \multicolumn{3}{|c|}{ Approach 3} & \multicolumn{3}{|c|}{ Approach 4} \\
\hline & & & lane 1 & lane 2 & lane 3 & lane 1 & lane 2 & lane 3 & lane 1 & lane 2 & lane 3 & lane 1 & lane 2 & lane 3 \\
\hline \multirow{3}{*}{ f4 } & \multirow{3}{*}{1} & A & 0.55 & 0.61 & 0.52 & 0.64 & 0.60 & 0.54 & 0.68 & 0.70 & 0.63 & 0.82 & 0.83 & 0.78 \\
\hline & & $\mathbf{Q}$ & 126.13 & 132.54 & 76.09 & 75.34 & 86.92 & 61.43 & 44.40 & 52.10 & 37.60 & 0.62 & 0.64 & 0.66 \\
\hline & & H & 0.55 & 0.60 & 0.51 & 0.64 & 0.62 & 0.55 & 0.68 & 0.70 & 0.64 & 0.82 & 0.83 & 0.78 \\
\hline
\end{tabular}




\begin{tabular}{|c|c|c|c|c|c|c|c|c|c|c|c|c|c|}
\hline & $\mathbf{R}$ & 126.39 & 131.97 & 75.56 & 75.80 & 89.00 & 61.55 & 44.40 & 52.10 & 38.13 & 0.62 & 0.64 & 0.66 \\
\hline \multirow{4}{*}{2} & $\mathbf{A}$ & 0.52 & 0.51 & 0.48 & 0.58 & 0.55 & 0.49 & 0.66 & 0.69 & 0.62 & 0.82 & 0.83 & 0.74 \\
\hline & $\mathbf{Q}$ & 130.33 & 155.47 & 80.15 & 82.60 & 93.74 & 65.26 & 47.83 & 52.11 & 37.95 & 0.64 & 0.65 & 0.78 \\
\hline & $\mathbf{H}$ & 0.52 & 0.50 & 0.48 & 0.59 & 0.56 & 0.49 & 0.65 & 0.70 & 0.63 & 0.81 & 0.82 & 0.73 \\
\hline & $\mathbf{R}$ & 131.15 & 154.75 & 80.24 & 83.84 & 96.55 & 65.97 & 47.26 & 52.57 & 38.62 & 0.64 & 0.64 & 0.77 \\
\hline \multirow{4}{*}{3} & A & 0.52 & 0.55 & 0.49 & 0.51 & 0.55 & 0.52 & 0.64 & 0.72 & 0.63 & 0.80 & 0.82 & 0.75 \\
\hline & $\mathbf{Q}$ & 130.45 & 146.14 & 79.03 & 91.35 & 94.10 & 62.08 & 49.86 & 47.54 & 36.49 & 0.72 & 0.66 & 0.77 \\
\hline & $\mathbf{H}$ & 0.52 & 0.55 & 0.49 & 0.52 & 0.56 & 0.53 & 0.63 & 0.73 & 0.65 & 0.79 & 0.82 & 0.73 \\
\hline & $\mathbf{R}$ & 131.34 & 145.51 & 79.29 & 92.75 & 96.92 & 62.76 & 49.27 & 47.98 & 37.66 & 0.70 & 0.66 & 0.76 \\
\hline \multirow{4}{*}{4} & $\mathbf{A}$ & 0.53 & 0.56 & 0.48 & 0.53 & 0.61 & 0.49 & 0.62 & 0.71 & 0.60 & 0.79 & 0.82 & 0.74 \\
\hline & $\mathbf{Q}$ & 128.66 & 144.16 & 80.54 & 88.58 & 84.92 & 63.13 & 49.92 & 48.65 & 38.33 & 0.74 & 0.67 & 0.76 \\
\hline & $\mathbf{H}$ & 0.53 & 0.56 & 0.48 & 0.54 & 0.62 & 0.51 & 0.63 & 0.72 & 0.62 & 0.78 & 0.82 & 0.74 \\
\hline & $\mathbf{R}$ & 129.32 & 143.64 & 79.61 & 91.22 & 87.48 & 65.23 & 50.47 & 49.33 & 39.69 & 0.73 & 0.67 & 0.76 \\
\hline \multirow{4}{*}{5} & $\mathbf{A}$ & 0.51 & 0.51 & 0.50 & 0.58 & 0.55 & 0.51 & 0.65 & 0.71 & 0.63 & 0.79 & 0.81 & 0.74 \\
\hline & $\mathbf{Q}$ & 129.84 & 156.39 & 79.19 & 80.13 & 93.72 & 61.47 & 46.90 & 49.80 & 36.31 & 0.74 & 0.68 & 0.76 \\
\hline & $\mathbf{H}$ & 0.52 & 0.50 & 0.49 & 0.60 & 0.57 & 0.53 & 0.66 & 0.71 & 0.65 & 0.78 & 0.81 & 0.74 \\
\hline & $\mathbf{R}$ & 132.00 & 155.09 & 77.62 & 82.97 & 97.08 & 64.11 & 47.49 & 50.20 & 37.62 & 0.74 & 0.68 & 0.76 \\
\hline
\end{tabular}

\begin{tabular}{|c|c|c|c|c|c|c|c|c|c|c|c|c|c|c|}
\hline \multirow{2}{*}{$V$} & \multirow{2}{*}{$x$} & & \multicolumn{3}{|c|}{ Approach 1} & \multicolumn{3}{|c|}{ Approach 2} & \multicolumn{3}{|c|}{ Approach 3} & \multicolumn{3}{|c|}{ Approach 4} \\
\hline & & & lane 1 & lane 2 & lane 3 & lane 1 & lane 2 & lane 3 & lane 1 & lane 2 & lane 3 & lane 1 & lane 2 & \begin{tabular}{|l|} 
lane 3 \\
\end{tabular} \\
\hline & \multirow{4}{*}{1} & $\mathbf{A}$ & 0.00 & 0.00 & 0.30 & 0.00 & 0.11 & 0.16 & 0.25 & 0.00 & 0.08 & 0.00 & 0.00 & 0.05 \\
\hline & & $\mathbf{Q}$ & 0.03 & 0.04 & 0.10 & 0.03 & 0.03 & 0.10 & 0.03 & 0.02 & 0.04 & 0.04 & 0.03 & 0.08 \\
\hline & & $\mathbf{H}$ & 0.00 & 0.00 & 0.30 & 0.00 & 0.11 & 0.16 & 0.25 & 0.00 & 0.08 & 0.00 & 0.00 & 0.05 \\
\hline & & $\mathbf{R}$ & 0.03 & 0.04 & 0.10 & 0.03 & 0.03 & 0.10 & 0.03 & 0.02 & 0.04 & 0.04 & 0.03 & 0.08 \\
\hline \multirow{8}{*}{$\mathbf{f 5}$} & \multirow{4}{*}{2} & $\mathbf{A}$ & 0.00 & 0.00 & 0.21 & 0.00 & 0.00 & 0.13 & 0.00 & 0.00 & 0.08 & 0.00 & 0.00 & 0.23 \\
\hline & & $\mathbf{Q}$ & 0.03 & 0.04 & 0.10 & 0.03 & 0.03 & 0.10 & 0.03 & 0.02 & 0.04 & 0.04 & 0.03 & 0.07 \\
\hline & & $\mathbf{H}$ & 0.00 & 0.00 & 0.22 & 0.00 & 0.00 & 0.13 & 0.00 & 0.00 & 0.08 & 0.00 & 0.00 & 0.23 \\
\hline & & $\mathbf{R}$ & 0.03 & 0.04 & 0.11 & 0.03 & 0.03 & 0.10 & 0.03 & 0.02 & 0.04 & 0.04 & 0.03 & 0.07 \\
\hline & \multirow{4}{*}{3} & $\mathbf{A}$ & 0.13 & 0.08 & 0.30 & 0.00 & 0.00 & 0.00 & 0.00 & 0.00 & 0.15 & 0.08 & 0.13 & 0.14 \\
\hline & & $\mathbf{Q}$ & 0.03 & 0.04 & 0.10 & 0.03 & 0.03 & 0.11 & 0.03 & 0.02 & 0.04 & 0.04 & 0.03 & 0.07 \\
\hline & & $\mathbf{H}$ & 0.13 & 0.09 & 0.31 & 0.00 & 0.00 & 0.00 & 0.00 & 0.00 & 0.15 & 0.08 & 0.13 & 0.14 \\
\hline & & $\mathbf{R}$ & 0.03 & 0.04 & 0.10 & 0.03 & 0.03 & 0.11 & 0.03 & 0.02 & 0.04 & 0.04 & 0.03 & 0.07 \\
\hline & \multirow{4}{*}{4} & $\mathbf{A}$ & 0.00 & 0.08 & 0.18 & 0.00 & 0.00 & 0.13 & 0.00 & 0.17 & 0.08 & 0.18 & 0.13 & 0.09 \\
\hline & & $\mathbf{Q}$ & 0.03 & 0.03 & 0.11 & 0.03 & 0.03 & 0.11 & 0.03 & 0.02 & 0.04 & 0.04 & 0.03 & 0.08 \\
\hline & & $\mathbf{H}$ & 0.00 & 0.10 & 0.19 & 0.00 & 0.00 & 0.13 & 0.00 & 0.14 & 0.08 & 0.17 & 0.13 & 0.09 \\
\hline & & $\mathbf{R}$ & 0.03 & 0.04 & 0.11 & 0.03 & 0.03 & 0.11 & 0.03 & 0.02 & 0.04 & 0.04 & 0.03 & 0.08 \\
\hline & \multirow{4}{*}{5} & $\mathbf{A}$ & 0.00 & 0.00 & 0.21 & 0.00 & 0.00 & 0.13 & 0.13 & 0.00 & 0.00 & 0.00 & 0.00 & 0.09 \\
\hline & & $\mathbf{Q}$ & 0.03 & 0.03 & 0.11 & 0.03 & 0.03 & 0.11 & 0.03 & 0.02 & 0.04 & 0.04 & 0.03 & 0.08 \\
\hline & & $\mathbf{H}$ & 0.00 & 0.00 & 0.22 & 0.00 & 0.00 & 0.13 & 0.13 & 0.00 & 0.00 & 0.00 & 0.00 & 0.09 \\
\hline & & $\mathbf{R}$ & 0.03 & 0.04 & 0.11 & 0.03 & 0.03 & 0.11 & 0.03 & 0.02 & 0.04 & 0.04 & 0.03 & 0.08 \\
\hline
\end{tabular}

